\title{
Genome assembly of the tayra (Eira barbara, Mustelidae) and comparative genomic analysis reveal adaptive genetic variation in the subfamily Guloninae
}

Lorena Derežanin ${ }^{1^{*}}$, Asta Blažytè2 ${ }^{2}$ Pavel Dobrynin ${ }^{3}$, David A. Duchêne ${ }^{4}$, José Horacio $\mathrm{Grau}^{5}$, Sungwon Jeon ${ }^{6}$, Sergei Kliver ${ }^{7}$, Klaus-Peter Koepfli, ${ }^{3,8,9}$, Dorina Meneghini ${ }^{1}$, Michaela Preick ${ }^{10}$, Andrey Tomarovsky ${ }^{3,7,11}$, Azamat Totikov ${ }^{3,7,11}$, Jörns Fickel ${ }^{1,10}$, Daniel W. Förster ${ }^{1}$

1 Leibniz Institute for Zoo and Wildlife Research (IZW), Alfred Kowalke Straße 17, 10315 Berlin, Germany 2 Department of Biomedical Engineering, College of Information and Biotechnology, Ulsan National Institute of Science and Technology (UNIST), Ulsan, 44919, Republic of Korea

3 Computer Technologies Laboratory, ITMO University, 49 Kronverkskiy Pr., 197101 Saint Petersburg, Russia

4 Center for Evolutionary Hologenomics, The GLOBE Institute, Faculty of Health and Medical Sciences, University of Copenhagen, Øster Farimagsgade 5, 1353 Copenhagen, Denmark

5 amedes Genetics, amedes Medizinische Dienstleistungen GmbH, Jägerstr. 61, 10117 Berlin, Germany

6 Clinomics Inc., Ulsan, 44919, Republic of Korea

7 Institute of Molecular and Cellular Biology, SB RAS, 8/2 Acad. Lavrentiev Ave., Novosibirsk 630090, Russia

8 Smithsonian-Mason School of Conservation, 1500 Remount Road, Front Royal, VA 22630, USA

9 Smithsonian Conservation Biology Institute, Center for Species Survival, National Zoological Park, 1500 Remount Road, Front Royal, VA 22630, USA

10 Institute for Biochemistry and Biology, Faculty of Mathematics and Natural Sciences, University of Potsdam, Karl-Liebknecht-Str. 24-25, 14476 Potsdam OT Golm, Germany

11 Novosibirsk State University, 1 Pirogova str., Novosibirsk, 630090, Russia

${ }^{*}$ Corresponding author, lorenaderezanin@gmail.com 


\begin{abstract}
Species of the mustelid subfamily Guloninae inhabit diverse habitats on multiple continents, and occupy a variety of ecological niches. They differ in feeding ecologies, reproductive strategies and morphological adaptations. To identify candidate loci associated with adaptations to their respective environments, we generated a de novo assembly of the tayra (Eira barbara), the earliest diverging species in the subfamily, and compared this with the genomes available for the wolverine (Gulo gulo) and the sable (Martes zibellina). Our comparative genomic analyses included searching for signs of positive selection, examining changes in gene family sizes, as well as searching for species-specific structural variants (SVs). Among candidate loci that appear to be associated with phenotypic traits, we observed many genes related to diet, body condition and reproduction. For the tayra, which has an atypical gulonine reproductive strategy of aseasonal breeding, we observe species-specific changes in many pregnancy-related genes. For the wolverine, a circumpolar hypercarnivore that must cope with seasonal food scarcity, we observed many specific changes in genes associated with diet and body condition. Despite restricting some of our analyses to single-copy orthologs present in all three study species, we observed many candidate loci that may be linked to species traits related to environment-specific challenges in their respective habitats.
\end{abstract}

\title{
Introduction
}

The Mustelidae are the most ecologically and taxonomically diverse family within the mammalian order Carnivora, representing a remarkable example of adaptive radiation among mammals that is rich with recent speciation events (Koepfli et al. 2008; Liu et al. 2020). Closely related mustelid species often inhabit vastly different ecosystems, where they experience diverse environmental challenges and are thus exposed to different evolutionary pressures. This is particularly pronounced in the mustelid subfamily Guloninae, within which species occupy a variety of ecological niches, ranging from scansorial omnivores in the neotropics to terrestrial hypercarnivores in circumpolar regions. Members of the Guloninae display a range of behavioural and physiological adaptations associated with environment-specific resource availability, and consequently differ markedly in feeding ecology, reproductive strategy and morphology (Zhou et al. 2011; Heldstab et al. 2018). Here, we focus on tayra, wolverine and sable (Figure 1), for which genomic resources are now available. 


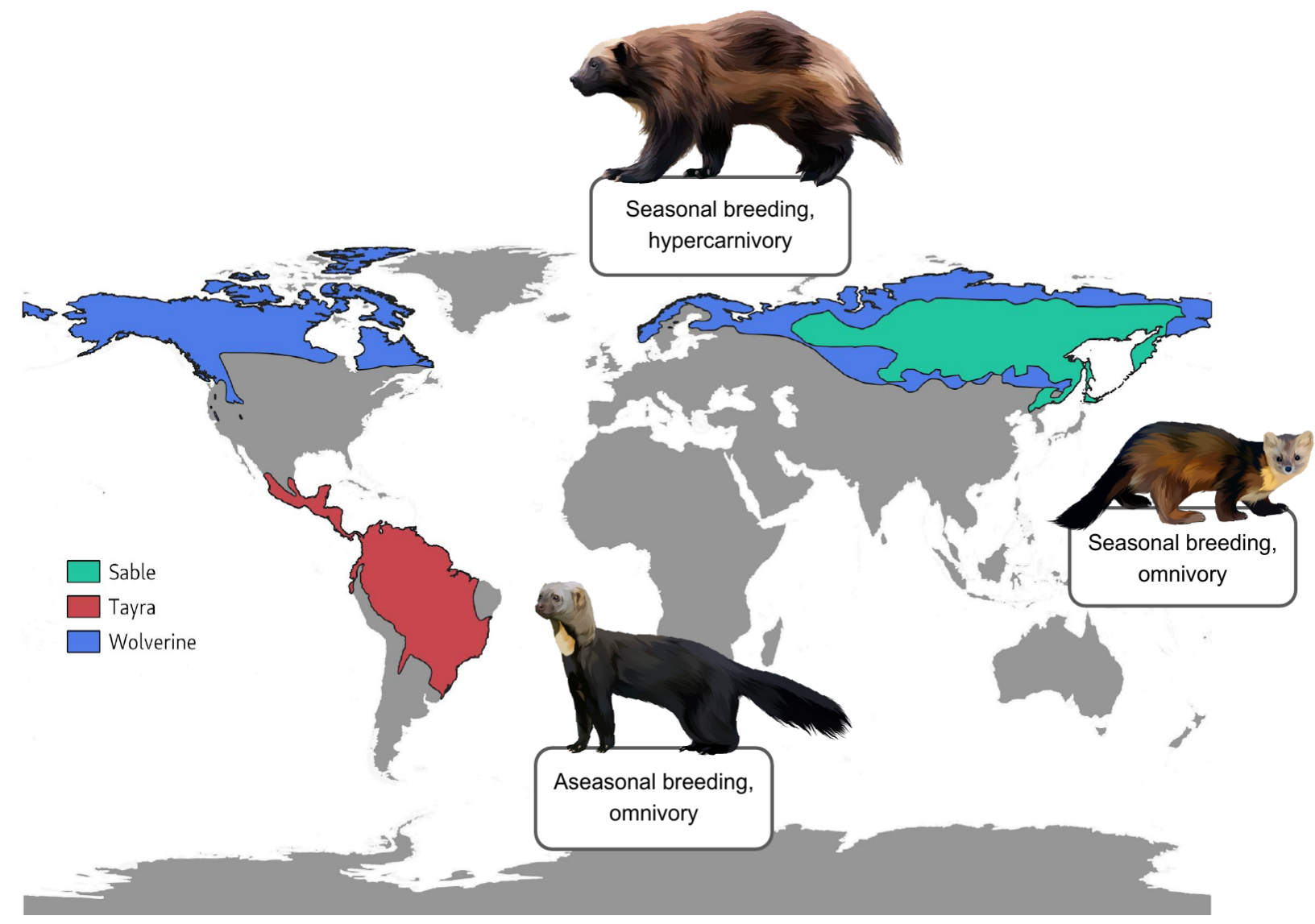

Figure 1. Distribution and species-specific traits of the tayra (Eira barbara), wolverine (Gulo gulo), and sable (Martes zibellina). Vector graphics of species are created based on royalty-free images (source: Shutterstock).

The tayra (Eira barbara) is a predominantly diurnal, solitary species that inhabits tropical and subtropical forests of Central and South America, ranging from Mexico to northern Argentina (Wilson and Mittermeier 2009). It is a scansorial, opportunistic omnivore, feeding on fruits, small mammals, birds, reptiles, invertebrates and carrion. Caching of unripe fruit for later consumption has been observed (Soley and Alvarado-Díaz 2011). Unlike other gulonine species, which are characterized by seasonal breeding and embryonic diapause, the tayra is an aseasonal polyestrous breeder and does not exhibit delayed implantation (Proulx and Aubry 2017), which may be due to the less prominent seasonality and fluctuation in food availability in neotropical habitats (Heldstab et al. 2018).

The largest terrestrial mustelid, the wolverine (Gulo gulo), is a circumpolar species, inhabiting alpine and boreal zones across North America and Eurasia (Ekblom et al. 2018). The wolverine is an opportunistic predator and facultative scavenger, either 
feeding on carrion or actively hunting medium to large-sized mammals, such as roe deer, wild sheep and occasionally moose (Pasitschniak-Arts and Larivière 1995). Morphological and behavioural adaptations such as dense fur, plantigrade locomotion facilitating movement through deep snow, and food caching, enable wolverines to survive in cold habitats with limited food resources (Copeland and Kucera 1997). In addition, wolverines occupy large home ranges, display territoriality, seasonal breeding and delayed implantation, traits indicating an adaptive response necessary for survival in scarce resource environments (Inman et al. 2012).

The sable (Martes zibellina) is distributed in the taiga and deciduous forests of north central and north eastern Eurasia. The sable is solitary and omnivorous, relying on hearing and olfaction to locate prey, even under a snow cover during winter months (Monakhov 2011; Liu et al. 2020). Unlike wolverines, seasonal changes do not cause dramatic fluctuations in resource availability for sables as they are able to exploit a wider variety of food sources, and are adapted to tolerate short-term food scarcity (Mustonen et al. 2006). Their diet consists of small mammals, birds, nuts and berries, and in some instances food caching during the winter period has been reported (Monakhov 2011). Similar to wolverines and many other species of mustelids, sables have a well-defined reproductive season and exhibit delayed blastocyst implantation (Proulx and Aubry 2017).

To date, only a few studies have investigated adaptive variation in mustelids using comparative genomics (Abduriyim et al. 2019; Beichman et al. 2019; Liu et al. 2020; Miranda et al. 2021). Here, we generated a highly contiguous genome assembly of the tayra, an early diverging gulonine (Koepfli et al. 2008; Law et al. 2018), and compared it to previously published genomes of the wolverine and sable to identify the genetic basis underlying the adaptations to the diverse environments inhabited by these species.

In addition to identifying genes under positive selection, we investigated gene family evolution and structural variants (SVs), as these types of variants represent a significant source of intra- and interspecific genomic differentiation, affecting more nucleotides than single-nucleotide polymorphisms (SNPs) (1000 Genomes Project Consortium et al. 2015; Catanach et al. 2019). Gene copy number variation and large SVs can be associated with an adaptive response to new ecological circumstances (e.g. diet in brown and polar bears (Rinker et al. 2019), and are thus an important source of genomic novelty to consider when studying adaptive divergence among species (Hecker et al. 2019). Interpretation of our results focuses on candidate loci linked to species-specific traits related to environment-specific challenges associated with resource availability in the respective habitats in each of the three species. 


\section{Results}

\section{Genome Assembly}

Using the 10x Genomics Chromium linked-read technology (Weisenfeld et al. 2017), we generated the first highly contiguous reference genome assembly for Eira barbara from genomic DNA obtained from a captive male tayra. The linked-read technology leverages long-range genomic information while maintaining the advantages of short-read sequencing on Illumina platforms (Armstrong et al. 2019). The extracted genomic DNA had an average molecule size of $50.75 \mathrm{~kb}$ and was sequenced at $\sim 76$-fold coverage (SI Table S1). The final assembly showed a total length of $\sim 2.44 \mathrm{~Gb}$ (excluding scaffolds shorter than $5 \mathrm{~kb}$ ), with a contig N50 size of $290 \mathrm{~kb}$ and a scaffold N50 size of $42.1 \mathrm{Mb}$. Whole genome alignment between tayra and domestic ferret assemblies shows identity in $95 \%$ of all positions (SI Figure S1). The tayra assembly has higher contiguity than the Illumina-only based assemblies of both wolverine and sable, but it is more fragmented than the chromosome-length domestic ferret (Mustela putorius furo) assembly we used as reference genome (Table 1, SI Figure S2A). The haploid genome size of $\sim 2.4 \mathrm{~Gb}$ is contained in 162 scaffolds (>100 kb) with 40 scaffolds having lengths above $50 \mathrm{Mb}$ (SI Figure S2A).

The tayra assembly has high gene completeness as assessed with BUSCO v4.1.2 using 9226 conserved mammalian orthologs (Simão et al. 2015). In total, 8540 (92.5\%) complete Benchmarking Universal Single-Copy Orthologs (BUSCOs) were identified, encompassing $8492(92.0 \%)$ of complete and single-copy, and $48(0.5 \%)$ complete and duplicated orthologs. Additionally, 104 (1.1\%) orthologs were fragmented and 582 $(6.4 \%)$ were missing. As measured by this metric, the presented tayra genome has higher gene completeness than the published genomes of wolverine, sable or domestic ferret (SI Figure S2B). 
Table 1. Comparison of genome assembly metrics among four mustelid species.

\begin{tabular}{|c|c|c|c|c|}
\hline Species & $\begin{array}{c}\text { Tayra } \\
\text { (Eira barbara) }\end{array}$ & $\begin{array}{l}\text { Domestic ferret } \\
\text { (M. putorius furo) }\end{array}$ & $\begin{array}{c}\text { Sable } \\
\text { (Martes zibellina) }\end{array}$ & $\begin{array}{l}\text { Wolverine } \\
\text { (Gulo gulo) }\end{array}$ \\
\hline $\begin{array}{l}\text { Assembly } \\
\text { Accession/ } \\
\text { reference }\end{array}$ & $\begin{array}{l}\text { JAHRIG000000000 } \\
\text { (This study) }\end{array}$ & $\begin{array}{l}\text { DNA Zoo } \\
\text { (Dudchenko et al. } \\
\text { 2018: Dudchenko } \\
\text { et al. 2017: Peng et } \\
\text { al. 2014) }\end{array}$ & $\begin{array}{l}\text { GCA_012583365.1 } \\
\text { (Liu et al. 2020) }\end{array}$ & $\begin{array}{l}\text { GCA_900006375.2 } \\
\text { (Ekblom et al. 2018) }\end{array}$ \\
\hline $\begin{array}{l}\text { Sequencing/ } \\
\text { assembly } \\
\text { approach }\end{array}$ & $\begin{array}{l}\text { Illumina + } \\
\text { 10xGenomics/ } \\
\text { Supernova }\end{array}$ & $\begin{array}{l}\text { Illumina/ } \\
\text { ALLPATHS-LG + } \\
\text { Hi-C scaffolding }\end{array}$ & $\begin{array}{l}\text { Illumina/ } \\
\text { SOAPdenovo2 }\end{array}$ & $\begin{array}{l}\text { Illumina/ } \\
\text { SOAPdenovo }\end{array}$ \\
\hline $\begin{array}{l}\text { Raw } \\
\text { coverage }\end{array}$ & $75.6 x$ & $162 x$ & $114.5 x$ & $76 x$ \\
\hline Contig N50 & $289.9 \mathrm{~kb}$ & $44.7 \mathrm{~kb}$ & $41.7 \mathrm{~kb}$ & $3.6 \mathrm{~kb}$ \\
\hline $\begin{array}{l}\text { Scaffold } \\
\text { N50 }\end{array}$ & $42.0 \mathrm{Mb}$ & $145.3 \mathrm{Mb}$ & $5.2 \mathrm{Mb}$ & $0.2 \mathrm{Mb}$ \\
\hline $\begin{array}{l}\text { Number of } \\
\text { scaffolds }\end{array}$ & 14,579 & 7,428 & 15,814 & 47,417 \\
\hline $\begin{array}{l}\text { Total } \\
\text { genome } \\
\text { length }\end{array}$ & $2.44 \mathrm{~Gb}$ & $2.40 \mathrm{~Gb}$ & $2.42 \mathrm{~Gb}$ & $2.42 \mathrm{~Gb}$ \\
\hline
\end{tabular}

\section{Repetitive elements}

The 'repeat landscape' of the tayra assembly contains $\sim 0.85 \mathrm{~Gb}$ of repetitive elements (SI Table S2). L1 type LINE elements are the most abundant, constituting $23 \%$ of the tayra genome. L1 elements also show signs of recent proliferation in comparison to DNA transposons and LTR retroelements (SI Figure S3). Endogenous retroviruses constitute $3.8 \%$ of the tayra genome and can be classified as Gammaretroviruses and Betaretroviruses.

The overall 'repeat landscape' of the tayra genome assembly is comparable to other carnivore genomes (C. Peng et al. 2018; G. Liu et al. 2020). It is similar to that of the sable genome, differing mostly in the number of L1 LINE elements, which have been recently proliferating and accumulating within the tayra genome more than in other Guloninae genomes. The diversity of endogenous retroviruses is similar to other mustelids. Although endogenous delta-retroviruses have been described from a broad 
range of mammal genomes, including several smaller carnivores like mongoose (family Herpestidae) and the fossa (Cryptoprocta ferox) (Hron et al. 2019), no delta-retroviruses were found in the genome of tayra.

\section{Demographic reconstruction}

The reconstruction of historical demography from whole genome data for tayra and sable using the Pairwise Sequentially Markovian Coalescent model (PSMC; Li and Durbin 2011) showed similar effective population sizes ( $\mathrm{Ne}$ ) during the last one million years of the Pleistocene, but started to differ around twenty thousand years ago, when the $\mathrm{Ne}$ of the sable increased compared with that of the tayra (SI Figure S4). PSMC analysis for the wolverine genome was in agreement with a previous study (Ekblom et al. 2018), also displaying low Ne. Similarly, low $\mathrm{Ne}$ was previously observed in the African cheetah genome, also characterized by very low genetic diversity (Dobrynin et al. 2015).

\section{Nucleotide diversity}

The tayra, sable and wolverine assemblies were generated using different approaches and differ significantly in contiguity (Table 1). To address this issue, we generated pseudochromosome assemblies for each species using the chromosome-length assembly of the domestic ferret as reference. The domestic ferret has more chromosomes than the other species analyzed $(2 n=40$ vs. $2 n=38)$, and the same number (20) of pseudochromosomes (Lewin et al. 2019) were obtained after scaffolding in each case. For each assembly, we identified the $X$ chromosome (labeled as $p s \_c h r X$ ) and arranged pseudoautosomes (labeled as ps_aut1 - ps_aut19) according to the length of the original scaffolds in the domestic ferret reference. This allowed us to verify the sex of the animals using a coverage-based approach (SI Figure S5), which confirmed morphological sexing for the tayra (male) and wolverine (female) individuals.

Surprisingly, we found a discrepancy between the results of our genome-based sexing for sable and the original results published with the sable genome (Liu et al. 2020). There, the sable individual is referred to as a male, our analysis suggests it is a female. Its $p s \_c h r X$ had uniform full (relative) coverage $(0.75 x-1.25 x$ relative to median whole genome coverage, SI Figure S5B), similar to the wolverine individual (SI Figure S5C). In contrast, we observed a clearly visible pseudoautosomal region (PAR) in the male tayra individual (SI Figure S5A), which had full (relative) coverage, whereas the rest of 
ps_chrX had half coverage. Additional verification using the SRY protein gene sequence as a Y-specific marker confirmed this result. The orthologous full-length CDS of SRY (Supplementary files 1 and 2) was detected only in the tayra assembly, while only partial hits with low similarity were observed in the two other assemblies. This provides evidence that the sable individual is not male but female, and also neither XXY (Klinefelter syndrome in humans; Wikström and Dunkel 2011) nor XX with translocation of the SRY locus to the $X$ chromosome (de la Chapelle syndrome in humans; De la Chapelle et al. 1972).

We counted heterozygous SNPs in 1 Mbp stacking windows for all three species and scaled it to SNPs per kbp (Figure 2). Median values for tayra, sable and wolverine were 1.89, 1.44 and 0.28 SNPs per kbp, respectively, the latter being in agreement with previous findings (Ekblom et al. 2018). All scaffolds of $\geq 1 \mathrm{Mbp}$ in pseudochromosome assemblies were taken into account. Exclusion of ps_chrX resulted in slight increases of medians to $1.93,1.47$ and 0.29 , respectively (SI Table S3, SI Figure S6). The finding of similar patterns in the male tayra and the female sable and wolverine is explained by two different facts. First, in males most of the $X$ chromosome is hemizygous, and heterozygous variants in this region arise from artefacts of alignment and variant calling. Second, in females the $X$ chromosome is diploid, but diversity in the same region of $X$ chromosome that is hemizygous in males, is lower than in autosomes (A) even if the X/A ratio in the population is taken into account (Kondo and Ueda 2013). The PAR demonstrates the opposite pattern, but it is too short to significantly affect mean and median values (Filatov and Gerrard 2003). Regardless of whether the ps_chrX was included or excluded, the genome-wide diversities among the three species were significantly different ( $p$-values $<<0.001$, Mann-Whitney test). 
bioRxiv preprint doi: https://doi.org/10.1101/2021.09.27.461651; this version posted October 7, 2021. The copyright holder for this preprint (which was not certified by peer review) is the author/funder, who has granted bioRxiv a license to display the preprint in perpetuity. It is made available under aCC-BY-NC-ND 4.0 International license.

A

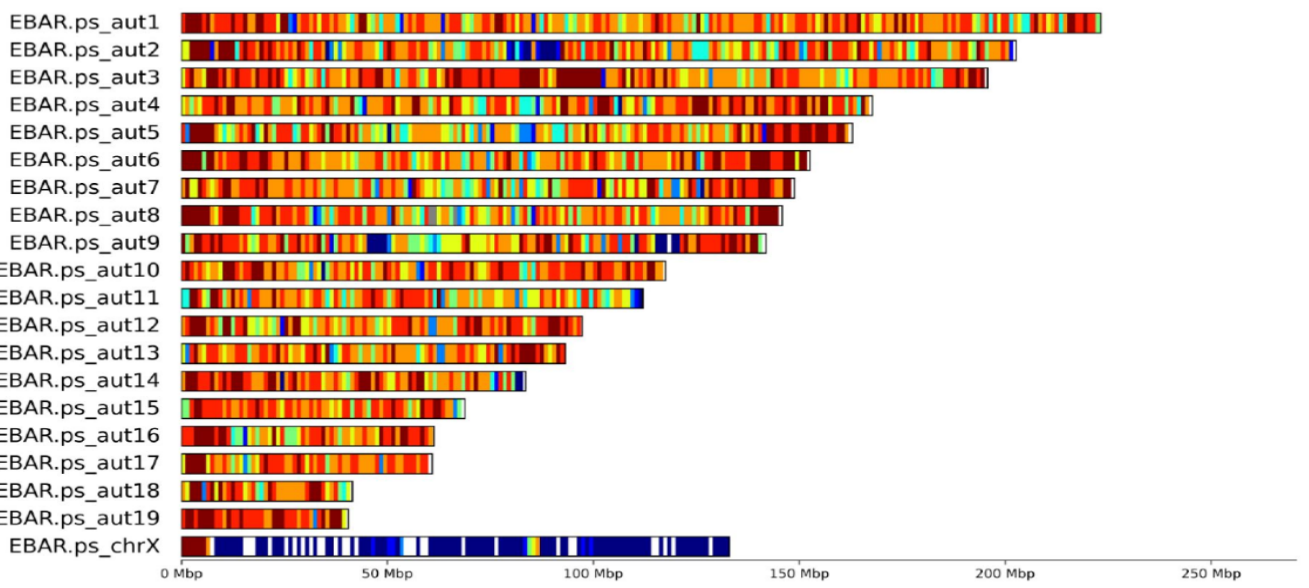

B

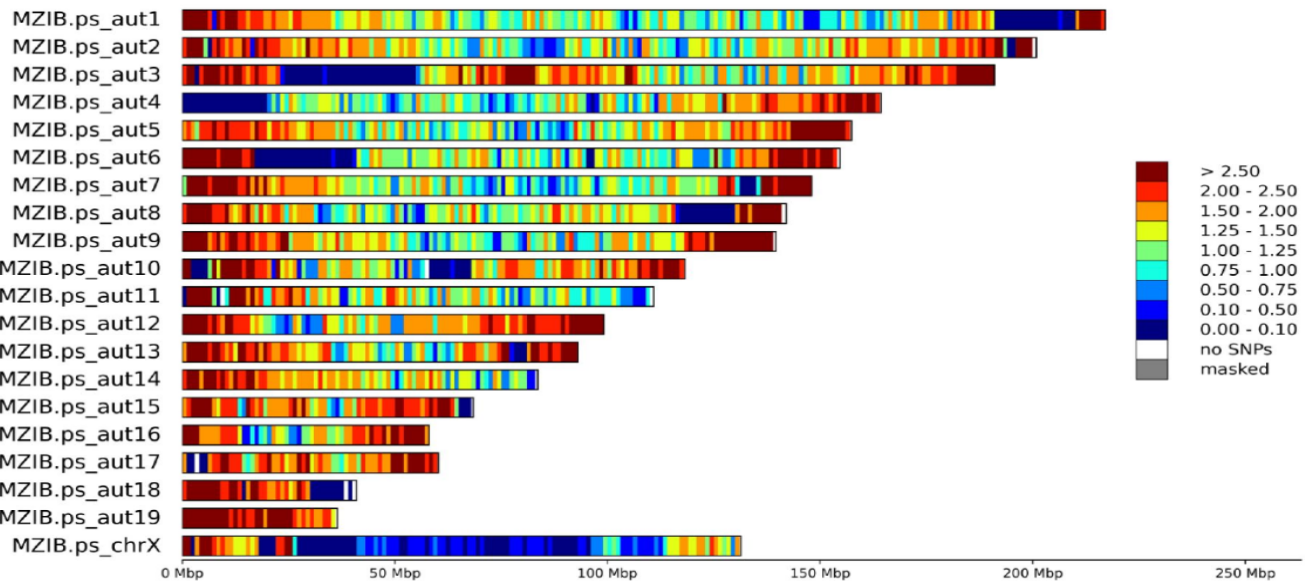

C

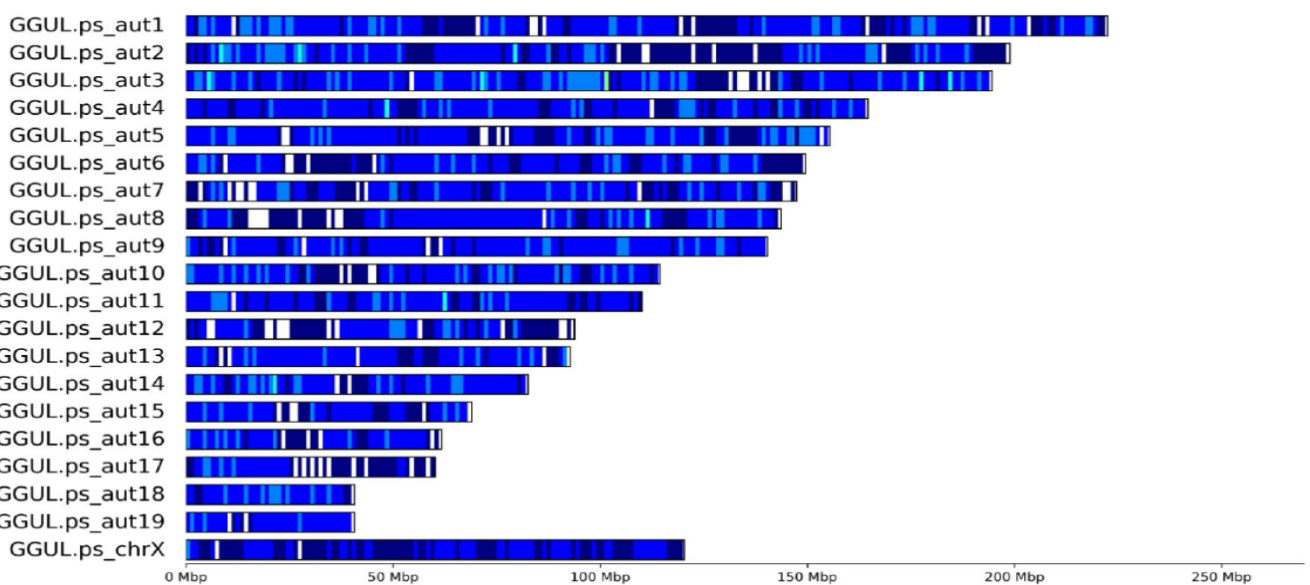

Figure 2. Heterozygosity density among pseudochromosomes for (A) tayra, (B) sable and (C) wolverine. Heterozygous SNPs were counted in stacking windows of $1 \mathrm{Mbp}$ and scaled to SNPs per kbp. Tayra is a 
male individual and thus heterozygous SNP density is underestimated (due to only one X chromosome), while sable and wolverine are females and therefore likely representative of true SNP density.

\section{Phylogenomics and molecular dating}

We reconstructed the phylogenetic relationships using single-copy orthologous genes from the BUSCO genome completeness analysis for assemblies of the following carnivoran species: domestic cat, domestic dog, walrus, northern elephant seal, domestic ferret, wolverine, sable, and tayra. Sequence alignment, cleaning and model adequacy assessment led to a phylogenomic data set with 2457 gene regions comprising over 3.2 million nucleotide sites. Phylogenomic analyses using concatenation and summary coalescent methods led to an identical resolution of the relationships among mustelid taxa. Within Guloninae, the wolverine and sable were placed as sisters, to the exclusion of the tayra (Figure 3). Branch supports were maximal across all branches using both approximate likelihood-ratio test (aLRT) and local posterior probabilities. Similarly, concordance factors (CF) for genes and sites (gCF, SCF) were high across branches and consistently more than twice as high as the values of the discordance factors (gDF, sDF). The lowest concordance factors were those in support of the resolution of Gulo and Martes as sisters (gCF $=64.52, \mathrm{sCF}=$ 54.38). However, the discordance factors were less than half these values (gDF $<14$, sCF < 24), suggesting substantial decisiveness across genes and sites for this resolution. 


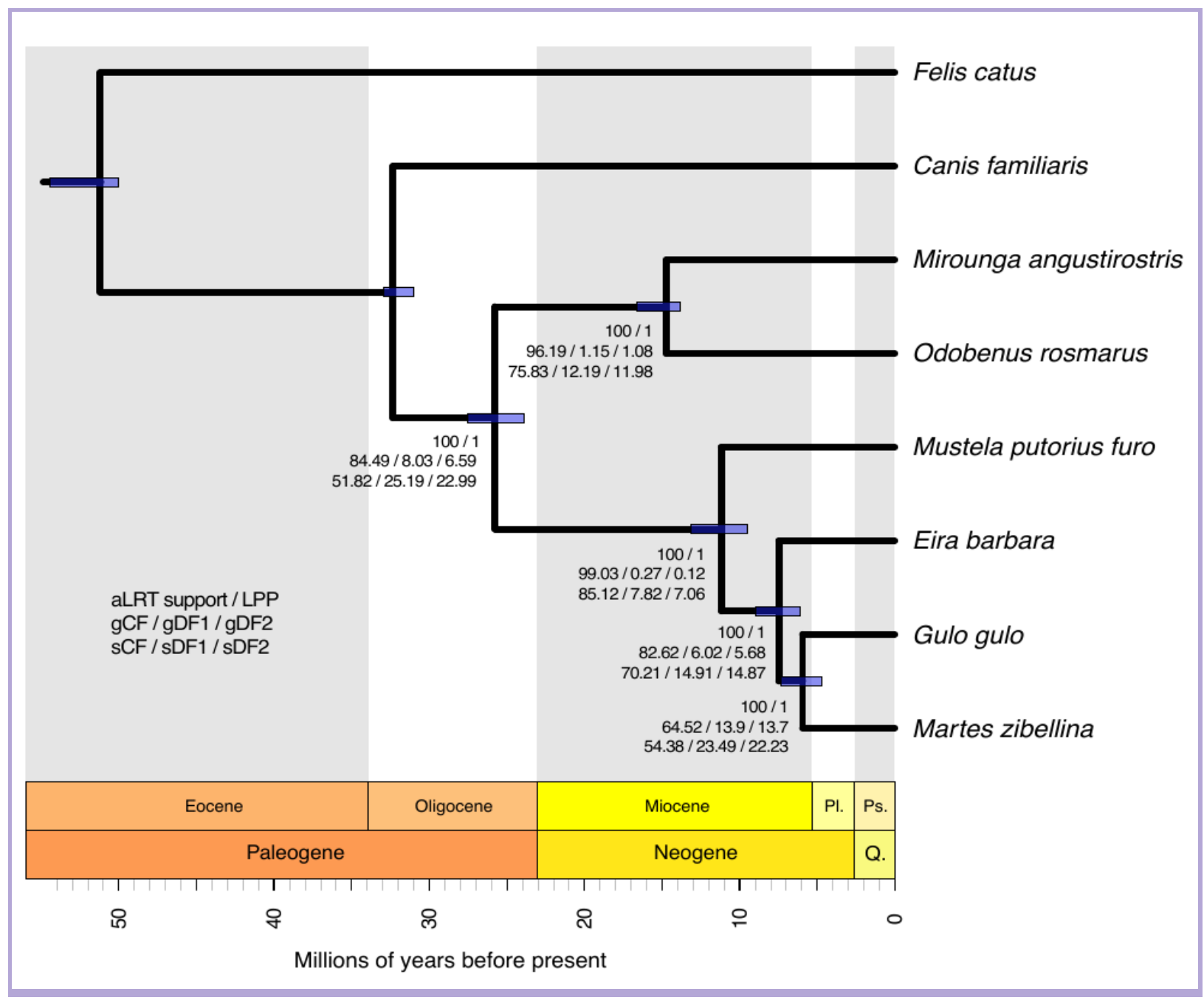

Figure 3. Phylogenetic tree and divergence times of Guloninae and five other carnivorans. The mean age of each node is shown, with $95 \%$ confidence intervals depicted as purple bars. The gene and site concordance (gCF, sCF) and discordance (gDF, sDF) factors are given, along with the branch support for both approximate likelihood-ratio test (aLRT) and local posterior probabilities (LPP).

Molecular dating was performed using 992 single-copy orthologous gene regions (comprising 0.53 million sites). Divergence time estimates across mustelids were largely in agreement with previous findings (Koepfli et al. 2008; Sato et al. 2012; Li et al. 2014; Law et al. 2018), placing the split between Mustela and Guloninae at 11.2 million years ago (Mya) (highest posterior density interval (HPDI) between 13.1 and 9.5 Mya), and the split between Eira and the Gulo-Martes group at 7.5 Mya (HPDI between 9 and 6.1 Mya). The split between Gulo and Martes was dated at 5.9 Mya (HPDI between 7.4 and 4.7 Mya). 


\section{Positive selection on single-copy orthologs}

We searched for sites under positive selection in single-copy orthologs in the three Guloninae species and in the five carnivoran species (domestic dog, domestic cat, domestic ferret, walrus, northern elephant seal) also used for the phylogenomic inference, to identify candidate loci involved in adaptation of the tayra, sable and wolverine to their respective environments. The CODEML branch-site test for positive selection was run on 6003 single-copy orthologs present in all species, for three separate foreground branches: tayra, sable and wolverine. We found sites under positive selection ( $5>\mathrm{dN} / \mathrm{dS}$ ratio $>1$, Barnett et al. 2020) in 55 orthologs, which were highly significant (free-ratio > 2). Of these 55 positively selected genes (PSGs), 15 were observed in tayra, 22 in wolverine, and 18 in sable (Figure 4A-B; SI Table S3).

Among the 15 PSGs we detected in tayra, five are associated with reproduction (NSMCE1, ETV2, SPATA25, MUC15, and PIH1D2) with functions involving spermatogenesis, placenta and embryo development, and blood vessel morphogenesis (Shyu et al. 2007; Dong et al. 2014; Metzger et al. 2015; Singh et al. 2019; Gòdia et al. 2020). Among the remaining ten PSGs, HSPB6 is involved in vasodilation and muscle contraction (Dreiza et al. 2010), DERA is associated with environmental stressors, including exposure to toxins (Salleron et al. 2014), and uricase (UOX) is a liver enzyme involved in purine catabolism and regulation of fructose metabolism (Johnson et al. 2020). Three PSGs (IP6K3, MAGIX and FAM149B1) are found to be associated with the nervous system, synapse formation and structural plasticity, as well as motor skills and coordination (Feng and Zhang 2009; Crocco et al. 2016; Shaheen et al. 2019). Three further PSGs (DUSP19, TNLG2B, and LRRC4) are related to the immune system (Ye and Ting 2008; Premzl 2016; Xie et al. 2020) and HEMK1 regulates methylation processes (Ishizawa et al. 2008).

We detected 22 PSGs in wolverine, including six genes associated with energy production and conversion. Among them, ATP6VOB, KMO, and SLC16A4 are primarily involved in insulin level regulation, and the metabolism of carbohydrates and fatty acids (Sun-Wada et al. 2001; Pierre et al. 2007; Avnet et al. 2020). Three PSGs (OIP5, ZADH2 and MTPAP) are specifically associated with adipose tissue formation and intramuscular fat deposition (Xiao et al. 2006; Yu et al. 2013; Inoue et al. 2014). Additionally, we found three PSGs (NBR1, TMEM38B, PPP1R18) involved in selective autophagy as response to nutrient deprivation along with bone mass and density regulation, and resorption (Xu et al. 2015; Cabral et al. 2016; Matsubara et al. 2018). We also detected PSGs (DAB1, OPA1 and CTNS) linked to cognition, brain 
development and vision (Blume et al. 2017; Del Dotto et al. 2018; Browning et al. 2019). Several PSGs (BNIPL, IL18BP, CRNN) were associated with the immune system; (Im et al. 2002; Xie et al. 2004; Nelson et al. 2008) while three others (ANAPC7, RNF212B, IZUMO3) are involved in reproduction processes (Grayson and Civetta 2012; Reynolds et al. 2013; Wild et al. 2018) and USB1 and CLCN4 have a role in basal cell cycle processes (Picollo and Pusch 2005; Nomura et al. 2018). For the last two, CEP95 and FAM185A, it was not possible to associate a specific phenotypic trait.

Among the 18 PSGs detected in the sable, three (PRRT2, ATL2, SELENOI) are associated with locomotion, and coordination (Rismanchi et al. 2008; Horibata et al. 2018; Valente et al. 2019), and USP53 is associated with sensory perception and nervous system (Kazmierczak et al. 2015). Two PSGs, VEGFC, RASA1, are associated with blood vessel formation (Rauniyar et al. 2018; Chen et al. 2019), three (TTC4, ZBP1, CD247) with the immune system (Kuriakose and Kanneganti 2018; Shang et al. 2018; Ye et al. 2019) and three (IQUB, UBQLNL, MEIKIN) with reproduction (Shi et al. 2009; Bao et al. 2010; Kim et al. 2015). Several PSGs (EEF2KMT, DEUP1, ECD, IQCK) are associated with cell cycle processes (Bähler and Rhoads 2002; Kim et al. 2010; Zhao et al. 2013; Davydova et al. 2014), and ZC2HC1C and CCDC17 could not be associated with a particular function or biological process.

\section{Gene family expansions and contractions}

Adaptive divergence between species may also be caused by changes in gene family sizes that occur during genome evolution and are due to gains (expansions) or losses (contractions) of genes or groups of genes (Olson 1999; Tigano et al. 2020). We analysed gene gains and losses while accounting for phylogenetic history by applying a probabilistic global birth and death rate model on gene sets containing 7838 orthologs present as either 'single-copy', 'duplicated' or 'missing' in at least one of the eight carnivoran species. Overall, the eight species analysed displayed more gene family contractions than expansions, with the wolverine having the highest contraction rate. This is most likely not a real biological finding but rather an artefact due to the fragmented genome assembly (SI Figure S7).

Tayra and sable had similar numbers of gene family expansions and contractions (SI Figure S7): 34 expansions and 169 contractions in tayra, and 33 expansions and 162 contractions in sable. The less contiguous wolverine genome contained 7 expansions and 649 contractions (SI Tables S4A, S4B). Due to the stochastic nature of gene losses 
and the potential inflation of estimates resulting from different genome assembly contiguities, we here focus on gains of gene copies.

Expanded gene families in the tayra genome are associated with cell cycle, metabolism, nervous and immune system, and reproduction, among others (Figure 4C-D; SI Table S4B). Of the three reproduction-related genes, SLC38A2 regulates supply of nutrients for fetal growth through the placenta and supports embryo development during the peri-implantation period of pregnancy (Coan et al. 2010; Forde et al. 2014). The second one, HSD17B10, is associated with regulation of pregnancy-sustaining steroid hormones (Martin Hill et al. 2010) while the RBP2, is involved in retinol binding and vitamin A metabolism necessary for oogenesis and embryogenesis (Brown et al. 2003) and also vision (D'Ambrosio et al. 2011). Three genes (ATP6V1D, DBX2, SLC38A1) are associated with cerebral cortex development, synapse formation, visual perception, and learning processes ( $\mathrm{Gu}$ et al. 2001). ANKRD13A is also associated with vision (like $R B P 2$ ), more specifically with lens fiber generation and vitamin A metabolism required for the formation of the photoreceptor rhodopsin (Avellino et al. 2013). The MAPKAPK5 gene is part of signalling pathways regulating fear conditioning and memory (Gerits et al. 2007) and N6AMT1 is involved in modulation of arsenic-induced toxicity (Zhang et al. 2015). The olfactory receptor gene TAAR5, specific for trimethylamine, a bacterial metabolite found in some animal odors, is involved in behavioral responses in mammals (Li et al. 2013; Liberles 2015) and was duplicated in both tayra and sable. We detected one putative triplication of FKBP3, a gene associated with immunoregulation, predominantly of T-cell proliferation (Kang et al. 2008). The PRR11 could not be associated with a particular function or biological process.

In the wolverine, two duplicated genes are related to the nervous system: GFRA4 is implicated in motor neuron development (Masure et al. 2000) and KCNS1 in regulating mechanical and thermal pain sensitivity (Tsantoulas et al. 2018). The MTM1 gene is associated with positive regulation of skeletal muscle tissue growth (Hnia et al. 2011) and MON1B is implicated in immune response to viral infection (Guo et al. 2006).

In the sable, expansions involve gene families associated with the nervous system, here specifically with sensory perception and locomotion, with angiogenesis, hair follicle development, and the immune system. The DUSP8 gene is involved in neuronal development, vision, olfaction, and spatial memory formation (Baumann et al. 2019; Martell et al. 1995). FBXL3 is associated with regulation of the circadian clock (Siepka et al. 2007) and TIMM10 with hearing (Paschen et al. 2000). Two genes, CDC42 and TCHHL1, are implicated in hair-follicle development (Wu et al. 2006; Yamakoshi et al. 2013). Additionally, CDC42, a gene coding for a cell division control protein, is also 
involved in angiogenesis and hematopoiesis, alongside TNFRSF12A and LXN (Wiley et al. 2001; Zhang and Liang 2018).

A

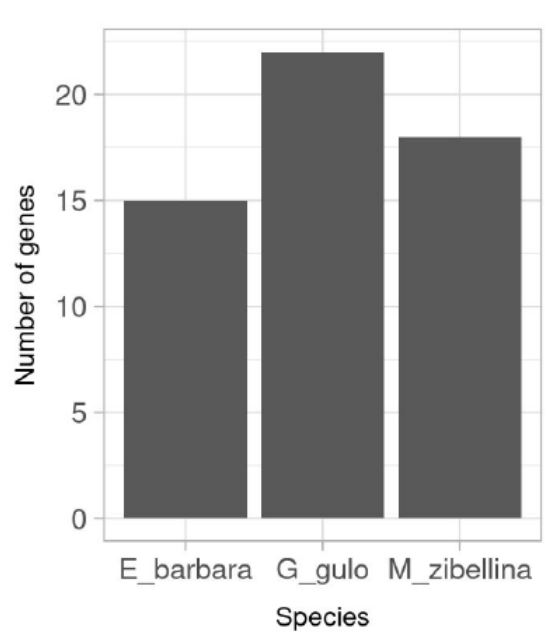

C

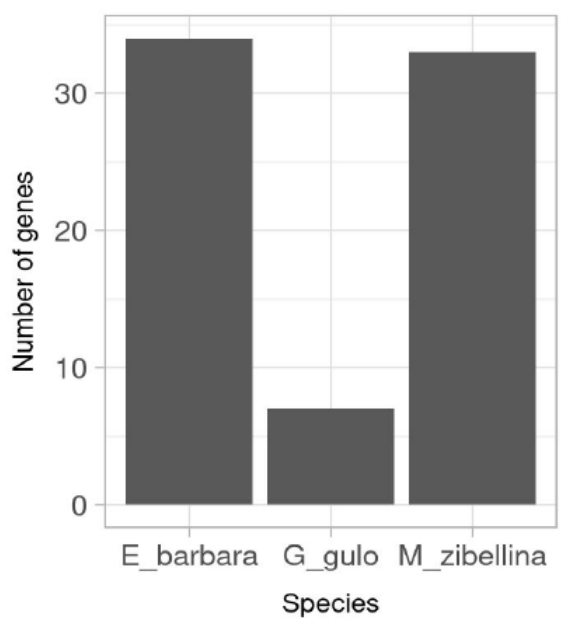

B

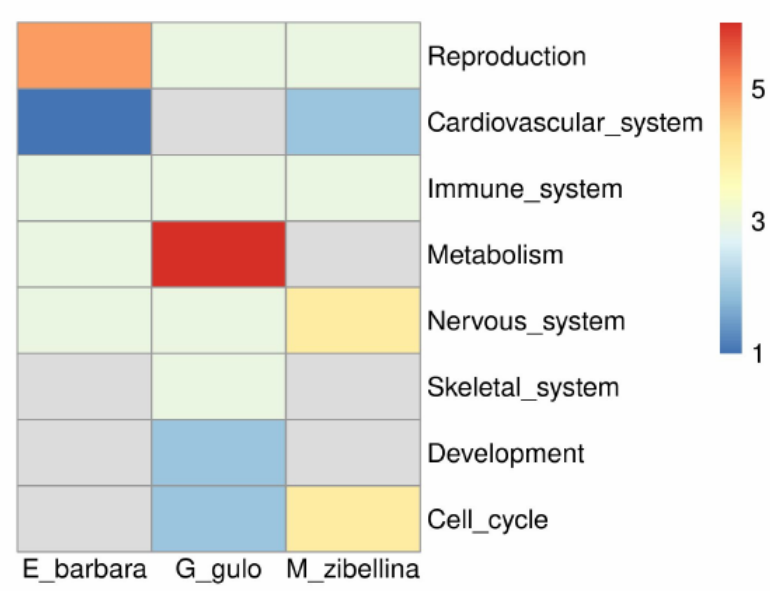

D

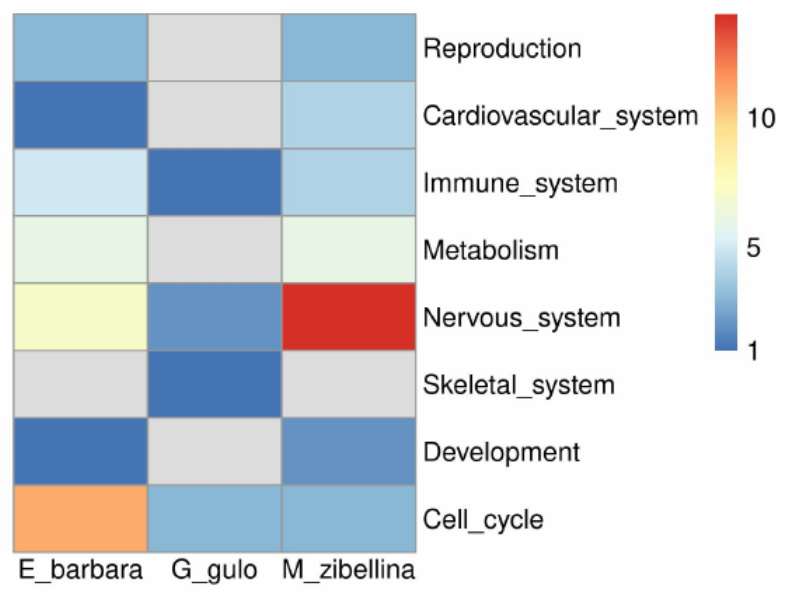

Figure 4. Number of candidate genes and their functional groups.

Genes identified from analyses of $(A, B)$ positive selection on single genes (PSG), and (C,D) gene family expansions. Heatmap scale indicates the number of genes.

\section{Structural variation}

Structural variants (SVs) modify the structure of chromosomes and can affect gene synteny, repertoire, copy number and/or composition (e.g. gain or loss of exons), create linkage-blocks, and modify gene expression (Chiang et al. 2017; Mérot et al. 2020), leading to complex variation in phenotypes and genetic diseases (Weischenfeldt et al. 2013). We investigated four types of SVs (deletions, duplications, insertions, inversions) 
in the three Guloninae species utilizing reads mapped to the domestic ferret genome and called with an ensemble of three SV callers (Kosugi et al. 2019).

We identified the highest number of species-specific SVs in sable (22979), followed by tayra (8907), and wolverine (264) (Figure 5A). The most abundant SVs detected in all three species are deletions (> 50bp), ranging from 183 species-specific deletions in wolverine to 21713 in sable. Duplications were the least frequent SV type among the three species (Figure 5B). For all three species, the majority of SVs are located in intergenic regions (> 80\%), with a smaller portion found in genic regions, completely or partially overlapping protein-coding genes (UTRs, exons, introns). According to Variant Effect Predictor (VEP) classification, SVs impacting genic regions are classified either as high-impact variants or modifiers (McLaren et al. 2016) with putative consequences on gene transcription ranging from transcript truncation to transcript ablation or amplification. The highest number of species-specific genic SVs is detected in tayra, with 330 (3.70\% of species-specific SVs), followed by $156(0.68 \%)$ in sable and 53 $(20.08 \%)$ in wolverine (Figure 5C). The exact impact of inversions overlapping large sets of genes is still challenging to determine, as the largest effect is likely to be restricted to genes near SV breakpoints, other than the well documented impact of inversions on intra and interspecific gene flow (Wellenreuther and Bernatchez 2018; Porubsky et al. 2020). Therefore, we further inspected genes and gene blocks affected by deletions, duplications, and insertions for their putative function and significantly enriched biological processes, respectively (Figure 5D, SI Table S5). 
A

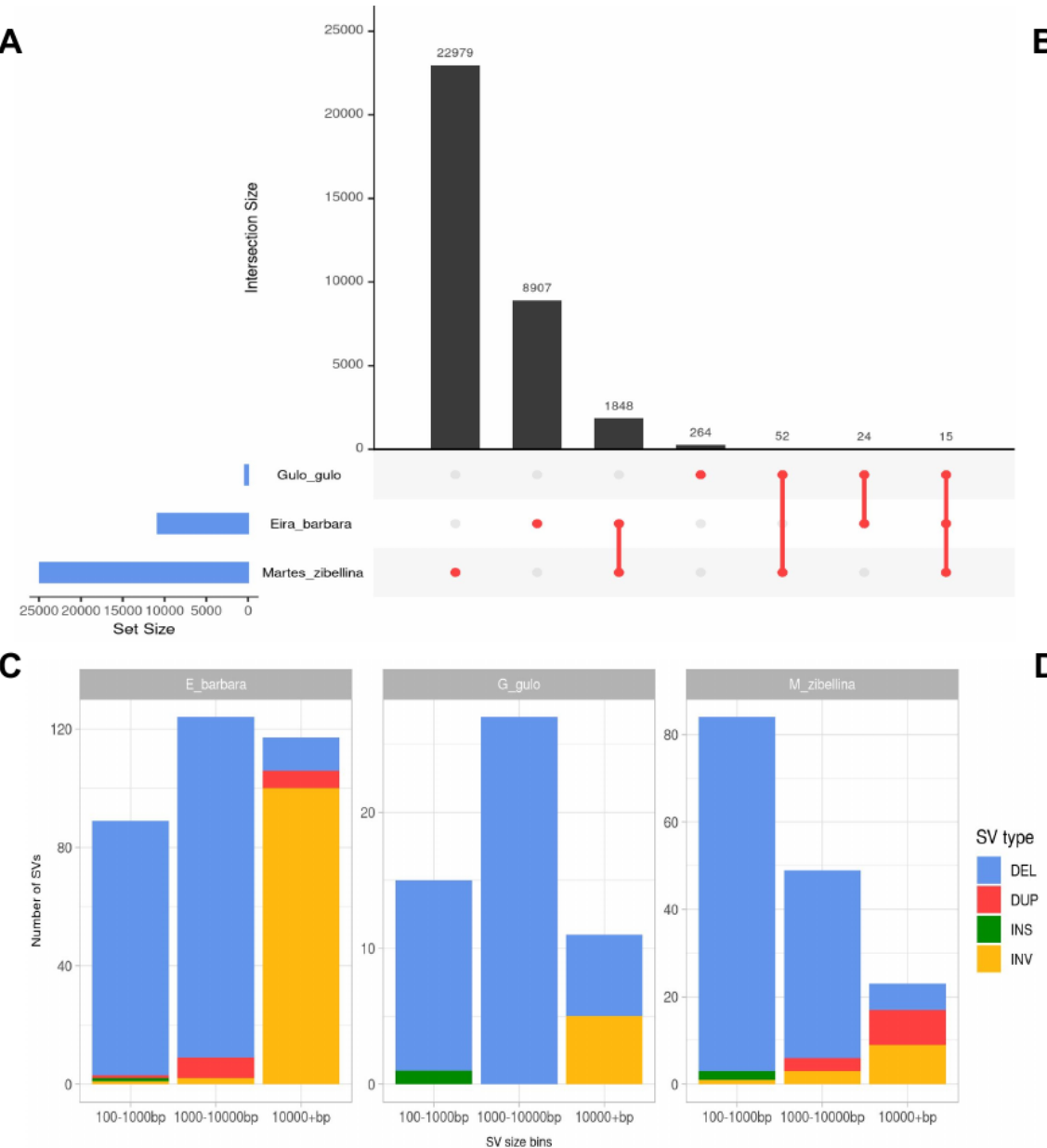

B

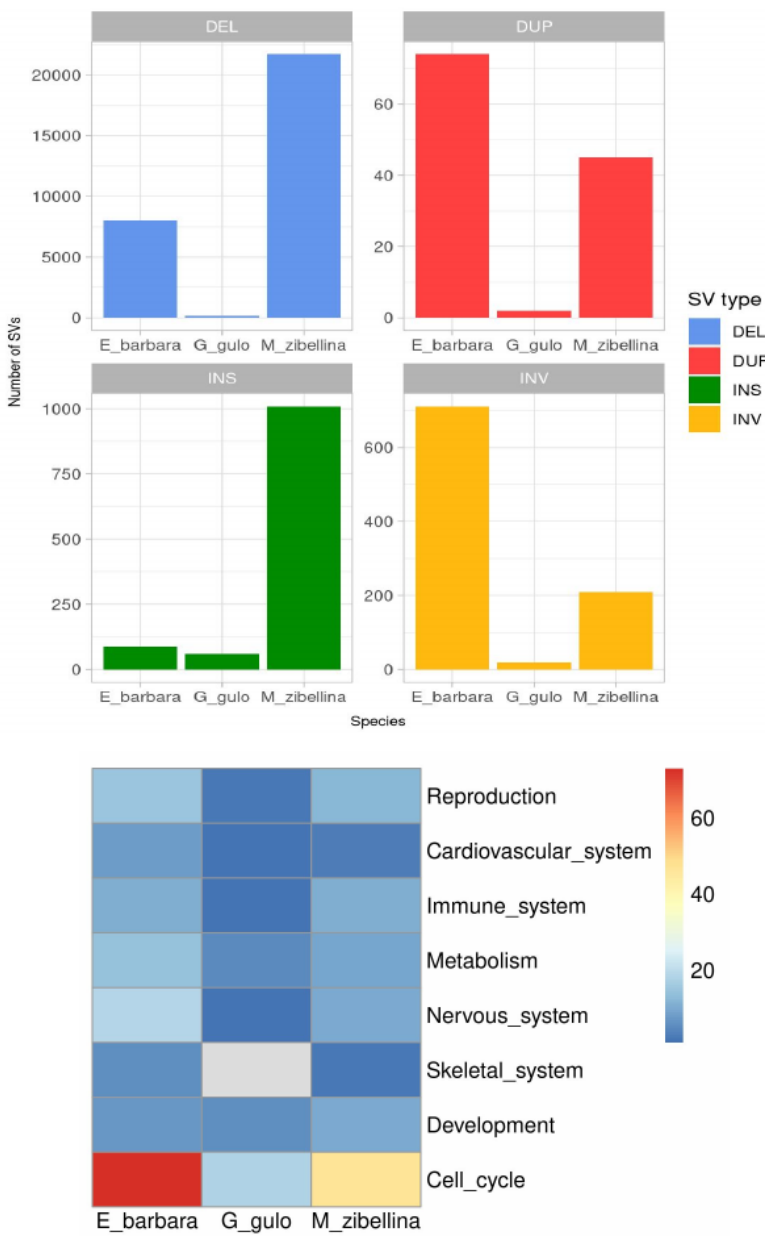

Figure 5. Structural variants detected in gulonine species.

A) Shared and species-specific structural variants detected in wolverine (Gulo gulo), tayra (Eira barbara), and sable (Martes zibellina), B) Counts of different types of species-specific structural variants detected in tayra (E_barbara), wolverine (G_gulo), and sable (M_zibellina), C) Length distribution of species-specific structural variants overlapping genic regions detected in tayra (E_barbara), wolverine (G_gulo), and sable (M_zibellina), D) Functional groups of genes affected by species-specific structural variants in 3 gulonine species (SV types: DEL, DUP, INS). Heatmap scale indicates the number of genes.

In the tayra genome, we observed 14 duplications spanning a combined length of 2.92 $\mathrm{Mb}$, putatively affecting 24 protein-coding genes. Duplicated genes and gene blocks are associated with olfaction, reproduction, metabolism and energy conversion. This included $R N A S E H 2 B$, a gene involved in in utero embryo development (Yockey and Iwasaki 2018), and two genes involved in spermatogenesis, DIAPH3 (Mironova and Millette 2008) and PCNX1 (Geisinger et al. 2005), the latter being an example of a complex SV involving heterozygous duplication and deletion of an exon (SV $\sim 2 \mathrm{~kb}$ in length). We detected 212 deletions in the tayra genome in relation to the domestic ferret reference, comprising a total length of $2.08 \mathrm{Mb}$, and affecting 247 genes, which are associated with cell cycle processes, metabolism/ energy conversion, nervous system, 
and reproduction, among other functional categories (SI Table S5). A majority of these genes are affected by partial (involving one or several exons) heterozygous deletions. Genes involved in placenta development and in utero embryogenesis include HSF1, RSPO2, and DNMT3A. Additionally, we detected NLRP1 and NLRP8, both associated with preimplantation development, highly expressed in oocytes, but with low to no expression in embryos (McDaniel and Wu 2009). One short insertion was observed in exon 2 of $L I X 1 L$, a gene associated with anatomical structure morphogenesis (Nakamura et al. 2015).

In the wolverine genome, none of the species-specific duplications overlapped genic regions. However, 47 deletions spanning a combined length of $229 \mathrm{~kb}$ are putatively associated with transcript truncation or ablation in 48 genes. The majority of affected genes are associated with basic cell cycle processes, development and metabolism/energy conversion. This includes GLUD1, a gene involved in amino-acid induced insulin secretion (Luczkowska et al. 2020), also found to be affected by a shorter deletion in sable, and $N S D H L$, a gene regulating cholesterol biosynthesis (Bauer et al. 2017). Additionally, we detected deletions affecting PARVA, a gene associated with angiogenesis and smooth muscle cell chemotaxis (Montanez et al. 2009) and DNAJC7, involved in positive regulation of ATPase activity and regulation of cellular response to heat (Sonna et al. 2002). We also detected one insertion in a gene of unknown function.

In the sable genome, we detected eleven duplications spanning a combined length of $324 \mathrm{~kb}$, overlapping 16 genes associated with cell cycle, sensory perception, development, and the immune system. The 130 detected deletions (combined length of $408 \mathrm{~kb}$ ) overlap 125 protein-coding genes associated with cell cycle, reproduction, the immune system, development, metabolism and sensory perception. Deletions were identified in two genes involved in keratinocyte differentiation, PPHLN1 (Larsen et al. 2017) and IVL, the latter of which is also associated with hair follicle development (de Viragh et al. 1994; Kitchener et al. 2018). Additionally, two short insertions were found disrupting exon 2 of NCOA4 and exon 7 of YIPF5, genes associated with mitochondrial iron homeostasis (hem synthesis) and protein transport, respectively.

\section{Discussion}

We present a highly contiguous genome for the tayra (Eira barbara), based on 10x Genomics linked-read data. Contiguity of the tayra genome assembly and its gene completeness are similar to or higher than those of other carnivoran species using the 
same technology (African wild dog: Armstrong et al. 2019, European polecat: Etherington et al. 2020, Southern elephant seal: Kim et al. 2020), confirming the utility of short linked-reads for genome assembly of wildlife species.

The phylogenomic relationships among the investigated mustelids were reconstructed using more than 900 orthologous CDS, and resulted in a tree topology and divergence time estimates in agreement with previous studies using fewer loci (Koepfli et al. 2008; Sato et al. 2012; Li et al. 2014; 2018). We estimated the split between Mustela and Guloninae occurred 11.2 million years ago (Mya) (HPDI 13.1 - 9.5 Mya), followed by the split between Eira and the Gulo-Martes group 7.5 Mya (HPDI 9 - 6.1 Mya), and the split between Gulo and Martes at 5.9 Mya (HPDI 7.4 - 4.7 Mya).

We did not observe a positive relationship between variation at the nucleotide level (heterozygous SNPs) and variation at the structural level (SVs) within the gulonine species. The tayra displayed the highest nucleotide diversity (1.89 SNPs per kbp, SI Table S3), but only the second highest amount of heterozygous SVs $(2543,23.6 \%$ of the total SVs, SI Figure S8). The sable had the second highest nucleotide diversity (1.44 SNPs per kbp), but the highest number of heterozygous SVs $(14823,59.5 \%$ of total). The wolverine displayed the lowest variation for both $(0.28$ SNPs per kbp, and 153 or $43.1 \%$ heterozygous SVs in total).

Assessment of variation among genome assemblies of closely related species strongly relies on the contiguity and completeness of analysed assemblies (Gurevich et al. 2013; Totikov et al. 2021). This needs to be accounted for when examining variation among discontiguous genome assemblies generated from short-read sequencing data. Here, the low contiguity of the wolverine assembly (Table 1; SI Figure S2) has likely impacted the number of PSGs and gene family expansions/contractions detected, and multiple, short insert size libraries sequenced at low coverage have likely resulted in lower SV detectability. However, even with the improved assembly contiguity, low diversity in the Scandinavian wolverine population is expected given previous results (Ekblom et al. 2018) and the species' historical demography (SI Figure S4).

For the sable, analysis of the heterozygosity distribution along chromosomes revealed that the ends of many pseudochromosomes had nearly double the heterozygosity than the median (> 2.5 SNPs per kbp vs median of 1.44 SNPs per kbp; Figure 2B, dark red color), while at least eight of the pseudochromosomes had very long stretches of low diversity (Figure 2B, blue color) that might be considered as runs of homozygosity (ROHs). Such distributed $\mathrm{ROHs}$ are often interpreted to be a consequence of recent inbreeding (Ceballos et al. 2018). This sable was sampled in the Greater Khingan mountains (Heilongjiang Province, China), very close to the edge of the species' range 
(Liu et al. 2020). The usual decline in abundance at the periphery coupled with partial isolation from the main part of the species' range might have resulted in recent inbreeding explaining the observed pattern. Such a positive relationship between intraspecific SV counts and census (population) size has also been demonstrated for other species (Weissensteiner et al. 2020). The sable displayed the highest historical effective population size of the three gulonines (SI Figure S4), which may account for the high number of SVs we detected in this species.

\section{Adaptive genomic variation}

Among positively selected genes, gene family expansions and coding regions impacted by SVs, we found numerous candidate loci related to species-specific traits in tayra, wolverine and sable. In particular, SVs featured genes related to traits of adaptive significance, strongly arguing for an inclusion of SV analysis in genome comparisons of wildlife species.

The three gulonine species differed with respect to which reproduction-related processes were associated with candidate genes. In the aseasonally breeding tayra, candidate genes detected in three analyses (PSGs, gene family expansions, SVs) were frequently pregnancy-related, while in the two seasonal breeders, sable and wolverine, candidate genes were involved in a greater diversity of reproduction-related processes, primarily in gametogenesis (Figure 6A, SI Tables S3, S4B, S5). This may reflect the transition from seasonal to aseasonal breeding in the tayra lineage, the only extant aseasonal breeder among the Guloninae.

Shifts in dietary preferences have been linked to positive selection in single genes (Kosiol et al. 2008) and to copy number variation in metabolism-related gene families in mammals (Hecker et al. 2019; Rinker et al. 2019). Among Guloninae, the dietary gradient from omnivory in the neotropics to (hyper)carnivory in the northern Palearctic is reflected in the proportion of metabolism-related candidate genes observed (Figure 6B). The highest proportion of candidate genes involved in carbohydrate metabolism ("omnivorous diet") were detected in tayra, followed by sable, and none detected in wolverine. However, the highest number of candidate genes involved in maintaining a stable body condition through shifts in energy sources (autophagy, fat storage and bone resorption) was observed for the wolverine, potentially reflecting this species' adaptive response to unfavourable environmental conditions in its circumpolar habitat. 
We note that in two analyses (positive selection and gene family evolution), our study did not consider the entire gene repertoire of these species but only variation in genes found as single-copy orthologs among mammalian species. Thus, the proportional differences observed in diet, body condition and reproduction related genes reported here are likely only an incomplete reflection of the genes involved in these traits.

A

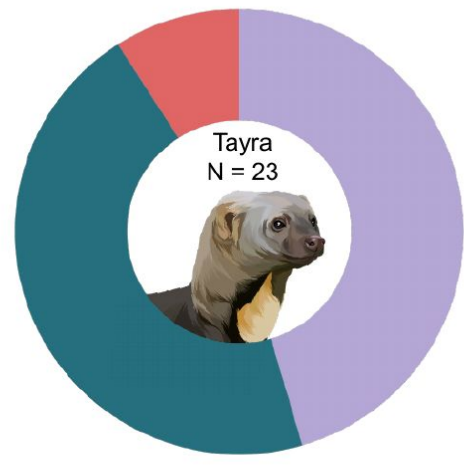

Seasonal breeding

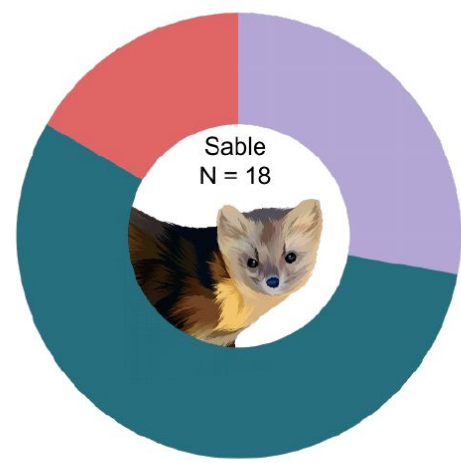

Seasonal breeding

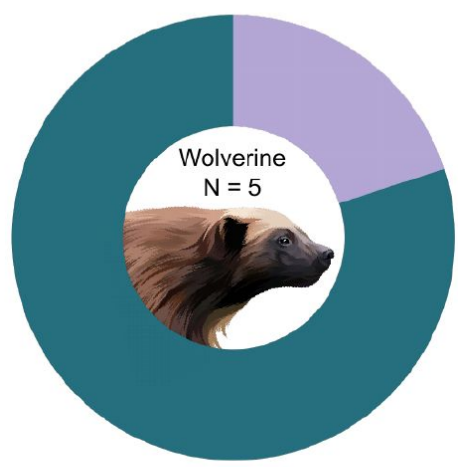

- Pregnancy Gametogenesis - Other (hormone regulation, milk secretion, circadian clock)

B

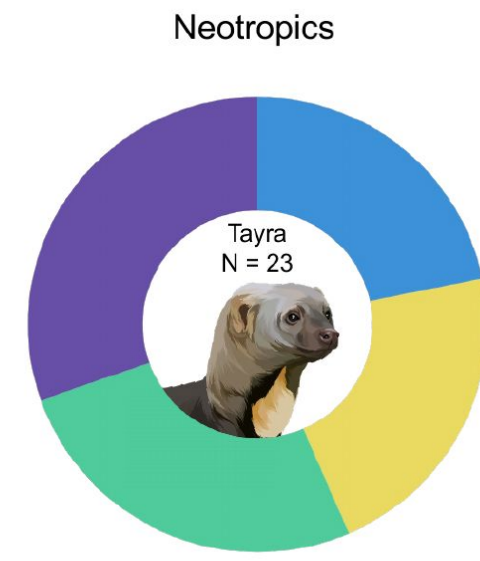

Northern Palearctic

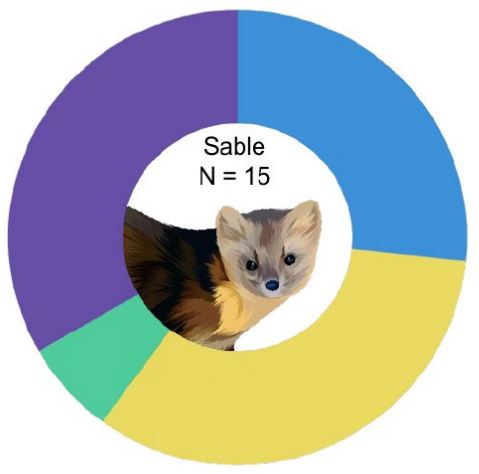

Northern Palearctic

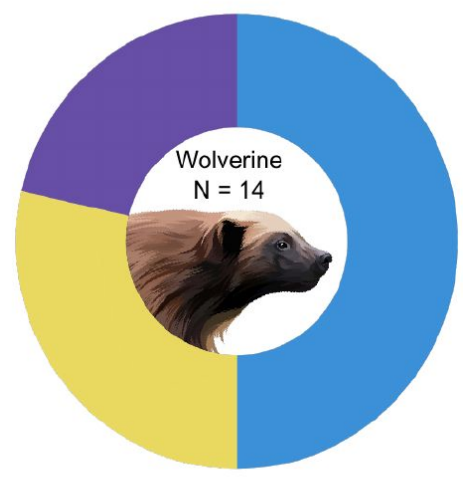

Body condition Carnivore diet Omnivore diet

Other (detoxification, insulin regulation, bile secretion, thermoregulation, glomerular permeability)

Figure 6. Summary of functional categories of $(A)$ reproduction and $(B)$ metabolism-related genes derived from PSG, gene family expansion and SV analyses. N represents the total number of detected genes. Vector graphics of species are created based on royalty-free images (source: Shutterstock). 


\section{Seasonal breeders in the north palaearctic: wolverine \& sable}

Obligate embryonic diapause or the delayed implantation of the blastocyst is a widespread reproductive strategy among mustelids and other carnivorans. The majority of mustelids in the Northern Hemisphere are seasonal breeders, which includes wolverines and sables that undergo a prolonged period of a delayed implantation lasting several months (Mead 1981; Svishcheva and Kashtanov 2011). This trait is also observed in fishers (P. pennanti) and martens (Mead 1989). Many environmental factors are associated with latitude, including photoperiod, temperature, seasonal changes in precipitation, all of which influence food abundance and availability. Optimal timing of ovulation and conception is crucial, and thus day length determines the onset of the breeding season in many species (Amstislavsky and Ternovskaya 2000). At higher latitudes, the mating season is even shorter, and there is a strong selection pressure for short birthing periods (Heldstab et al. 2018). As the breeding season represents one of the most energetically demanding periods, its timing in relation to when prey is abundant has a critical impact on the fitness of mustelids (Inman et al. 2012).

Candidate genes potentially involved in seasonal breeding that we detected in wolverine and sable are not restricted to those associated with pregnancy (SI Tables S3, S4B, S5). These mustelids occupy large home ranges, display territoriality and low population density, leading to infrequent encounters between males and females (Inman et al. 2012; Kashtanov et al. 2015). Thus, a mechanism that facilitates induced ovulation during the encounter would be an adaptive advantage (Larivière and Ferguson 2003). We detected signals of positive selection in ANAPC7 in wolverine, a gene involved in progesterone-mediated oocyte maturation and release from cell arrest prior to fertilization (Papin et al. 2004; Reis et al. 2006), that might have a role in increasing progesterone secretion and renewed embryonic development, as observed in skunks and mink (Mead 1989). As previously noted, delayed implantation serves as an important adaptation to ensure maximal survival chances of offspring depending on the environmental conditions (Heldstab et al. 2018). Previous studies in mink (Lopes et al. 2003; Murphy 2012) showed increased levels of vascular endothelial growth factors (VEGF) and their receptors, correlating with the implantation process. VEGFC, primarily associated with angiogenesis and regulation of permeability of blood vessels during embryogenesis, was positively selected in sable, suggesting its possible involvement in embryo implantation regulation in this species. 
Changes in testicular activity and spermatogenesis strongly correlate with season in mink (Blottner et al. 2006; Persson et al. 2011), and have been noted in lynx (Jewgenow et al. 2006), red deer (Pintus et al. 2015) and roe deer (Wagener et al. 2003). We observed spermatogenesis-related genes affected by different types of variation in the three gulonine species. In the wolverine, candidate genes showing signals of positive selection involved in spermatogenesis include IZUMO3 and RNF212B. Testes-expressed IZUMO3, a sperm membrane protein, is essential for gamete fusion during fertilization (Ellerman et al. 2009) and RNF212B is critical for successful crossing over in gametes. Knockout of RNF212 caused sterility in both male and female mice, with males not producing spermatocytes and having $70 \%$ smaller testes than those of wild-type animals (Reynolds et al. 2013). The IZUMO gene family most likely underwent expansion prior to the diversification of vertebrates, and several gene family members show signs of positive selection and indication of subfunctionalization (Grayson 2015). However, unlike other gene family members, positive selection of IZUMO3 is driven by a more recent mammalian-specific diversification and is suggested to be linked to species-specific adaptations rather than sexual selection (Grayson and Civetta 2012).

In sable, genes involved in spermatogenesis were UBQLNL (Marín 2014) and SEPT12, which show signals of rapid evolution, with the latter also being duplicated (i.e. gene family expansion). Moreover, MEIKIN and CDK2, both involved in meiosis, show signals of positive selection and rapid evolution through gene family expansion, respectively. MEIKIN has been found to be consistently rapidly evolving in other eutherian mammals (Pontremoli et al. 2021).

Seasonal breeding in many mammals is largely under photoperiod regulation, suggesting that the circadian system and pineal gland play an important role in measuring day length. Regulation of pineal melatonin secretion is influenced by a neural pathway that includes the circadian oscillators (Goldman 1999). Moreover, seasonal diapause is observed to be predominant among carnivores (Renfree and Fenelon 2017), and maintaining elevated melatonin in mink and spotted skunks has been shown to prolong embryonic diapause (Murphy and Fenelon 2020). FBXL3, associated with maintenance of circadian clock oscillation in mammals (Siepka et al. 2007; Shi et al. 2013; Fenelon et al. 2017) is duplicated and rapidly evolving in sable, indicating an important role in regulating both daily and seasonal activity and possibly onset of diapause. 


\section{Aseasonal breeder in the neotropic: tayra}

In tropical regions, reproductive seasonality is less pronounced as environmental conditions are relatively stable throughout the year (Dubost and Henry 2017; McNutt et al. 2019). Tayras inhabit tropical and subtropical forests of Central and South America, are aseasonal breeders with multiple estrous cycles per year (Proulx and Aubry 2017) and do not exhibit embryonic diapause (Poglayen-Neuwall et al. 1989). The tayra represents the most basal taxon of the Guloninae, and is an exception among these mustelids regarding its reproductive strategy.

Genes that may be involved in the aseasonal breeding of tayra include ETV2, MUC15, SLC38A2, HSD17B10, RBP2 and RNASEH2B. ETV2 and MUC15 are involved in placental and embryo development and regulation of embryo implantation (Shyu et al. 2007; Singh et al. 2019). The MUC15 protein has been shown to be present in the zona pellucida of preimplantation rat blastocyst, likely performing an anti-adhesive function, and preventing premature blastocyst attachment. Following the increase of progesterone levels, MUC15 levels are reduced, suggesting its removal may be required for a successful implantation (Denker 2000; Poon et al. 2014). Increased expression of the neutral amino acid transporter SLC38A2 has been detected in the endometrium of cattle during the preimplantation period of pregnancy (Forde et al. 2014) and in mice placentas during late gestation, maintaining fetal growth when maternal growth is restricted by undernutrition during pregnancy (Coan et al. 2010). SLC38A2 is a duplicated and rapidly evolving gene in tayra, based on our analysis, suggesting its potential role in supporting multiple pregnancies during the year. HSD17B10, involved in mitochondrial fatty acid metabolism, cell growth and cell resistance in response to stress (Liu et al. 2020) is highly expressed in the fetal and maternal liver, and has a role in metabolism of steroids that influence the maintenance of human pregnancy and provide protection against excitotoxicity following acute hypoxic stress (Hill et al. 2011). Duplication of this gene in tayra might indicate a gene dosage modulation in steroid hormone-regulated pregnancy in tayra. Beside being associated with vision, retinol binding protein $R B P 2$ has a significant role in regulating retinoids during oogenesis and embryogenesis, and positively impacts oocyte maturation in mice, cattle, pigs and sheep, leading to improved embryonic survival (Harney et al. 1993; Brown et al. 2003). A partial duplication was detected in RNASEH2B in tayra. Mutations in this gene involved in pregnancy and embryogenesis have been shown to cause interferonopathies, leading to inflammation disrupting fetal brain development (Crow et al. 2015; Yockey and Iwasaki 2018). In tayra, duplication of 
this gene potentially increases gene expression and may play a role in maintenance of early embryo development. Furthermore, genes involved in placental development, implantation and embryogenesis (HSF1, RSPO2, NLRP1,NLRP8 and DNMT3A) have been affected by partial deletions in tayra, suggesting further potential changes in dosage of pregnancy-related genes.

\section{Resource availability in the northern Palaearctic: wolverine \& sable}

Overcoming demanding periods and staying active throughout the year is challenging for non-hibernating northern palearctic species and requires specific mechanisms to cope with seasonal food scarcity. Responses to harsh environmental conditions aim to reduce the energy spent searching for food, and include physiological adaptations such as accumulation of fat reserves and adaptive fasting (Martinez and Ortiz 2017), as well as behavioural adaptations, like food caching (Mustonen et al. 2006) and flexible adjustment to alternative dietary sources (Zhou et al. 2011). For example, to successfully forage in a cold, low-productivity environment with scarce food resources, wolverines have evolved several adaptations to efficiently exploit food resources and overcome constraints of this niche (Inman et al. 2012). Wolverines both hunt and scavenge for the most energy-efficient food sources available in their habitat (Koskela et al. 2013). As it was demonstrated for female wolverines in Scandinavia, their reproduction is limited by food availability (Persson 2005). Scavenging from other predators such as gray wolf and Eurasian lynx facilitates food caching, leading to decreased unpredictability in food availability during winter and enhanced reproductive success of wolverines (Mattisson et al. 2017; Aronsson and Persson 2018).

A critical metric of body condition is the amount of stored fat, representing an important energy source between feeding opportunities and providing thermal insulation in cold environments. Undernutrition is a known cause affecting reproduction, and smaller energy reserves may lead to lower survival rates (Brown and Lasiewski 1972; Schulte-Hostedde et al. 2005). However, wolverines from Yukon, Canada, had no significant differences in fat deposits related to gender, season, body size or age although fat content was considerably higher in wolverines compared with other mustelids (Robitaille et al. 2012). Three PSGs detected in the wolverine are involved in adipose tissue formation, MTPAP (Han et al. 2012), OIP5 (Inoue et al. 2014), ZADH2 
(Yu et al. 2013) may be associated with lipid metabolism and selective fatty acid mobilization stimulated by fasting periods in this species (Krebs et al. 2004; Inman et al. 2012), as observed in mink (Nieminen et al. 2006), and raccoon dog (Mustonen et al. 2007).

One of the responses to prolonged periods of nutrient deprivation and extreme environmental conditions is suppressed bone resorption and formation, especially pronounced in hibernating species (Lennox and Goodship 2008; McGee-Lawrence et al. 2015). Beside regulating survival of blastocysts during delayed implantation (Lee et al. 2011; Lim and Song 2014), it has been demonstrated that autophagy plays a critical role in osteoclast differentiation and maintenance of bone homeostasis (DeSelm et al. 2011; Montaseri et al. 2020). This adaptation might be facilitated in wolverines by genes involved in bone mass regulation, including resorption (PPP1R18, TMEM38B) and autophagy (NBR1). Moreover, we detected a duplication of the muscle growth regulating gene MTM1. While a lack of MTM1 will lead to muscle hypertrophy through unbalanced autophagy in humans and mice (Al-Qusairi et al. 2013), a gene duplication may facilitate muscle growth.

In sable, fatty acids are uniformly mobilized from anatomically distinct fat deposits (Nieminen and Mustonen 2007), whereby the fatty acid metabolism is regulated by positively selected genes of the ACSL family, maintaining a low body fat content of $8 \%$ (Zhao et al. 2019). In our analyses we did not detect changes in the ACSL gene family, but we observed duplications of ASB6, SLC25A10, RNF186 and BORCS6, which have been implicated in effective regulation of fat storage usage and response to nutrient availability (Wilcox et al. 2004; Mizuarai et al. 2005; Schweitzer et al. 2015; Okamoto et al. 2020). Moreover, partial deletions of APOD, PDHB, LDLR, and CERS5, associated with lipid metabolic processes (Carmo et al. 2009; Serão et al. 2011; Tavori et al. 2015; Gosejacob et al. 2016), indicate a possible association with adaptive fasting and energy conservation.

We observed partial deletions in DNAJC7, a gene involved in thermoregulation and response to heat (Sonna et al. 2002) in both sable and wolverine, but not in tayra, indicating its potential significance for gulonines inhabiting colder environments. Furthermore, we detected partial deletions in GLUD1 in sable and wolverine. GLUD1 regulates insulin secretion (Fahien and Macdonald 2011) and might play a role in mobilizing lipids, causing intermittent states of reversible insulin resistance as an adaptive response during prolonged periods of nutrient deprivation, as has been observed in some carnivorans (Viscarra et al. 2013; Martinez and Ortiz 2017). 
In sable and tayra, we observed a partial deletion of PPP1CC, associated with glycogen metabolism ( $\mathrm{Li}$ et al. 2021), tentatively facilitating a more omnivorous diet in these species compared to hypercarnivory in wolverines (Sharma et al. 2018; Lopes-Marques et al. 2020).

Despite the notable difference in hair follicle structure and fur density between palearctic and neotropic species (Kitchener et al. 2018), we did not detect any type of variation affecting genes related to these traits in wolverine. However, we observed two gene copy gains in sable, TCHHL1, involved in hair morphogenesis (Wu et al. 2011), and CDC42, required for differentiation of hair follicle progenitor cells (Wu et al. 2006).

\section{Resource availability in the Neotropics: tayra}

Tayras exploit diverse food sources and experience relatively stable resource availability all year round, as (sub)tropical habitats have higher primary productivity compared to habitats at higher latitudes (Zhou et al. 2011; Heldstab et al. 2018). We found species-specific genes associated with regulation of fructose metabolism, consistent with dietary preferences of tayra involving a variety of fruit and honey (Kratzer et al. 2014). The uricase (UOX) gene, which is involved in regulation of purine metabolism and conversion of fructose to fat (Johnson and Andrews 2010) was under positive selection in tayra. Old World monkeys show low levels of the enzymatic activity while uricase is pseudogenized in humans, great apes, and New World monkeys due to silencing mutations (Oda et al. 2002; Johnson et al. 2009). Gradual loss of uricase activity in primates may have been an evolutionary adaptation to more rapidly convert fructose into fat storage during periods of progressively cooler climates.

High rates of lineage-specific variation in gene family size, especially those families involved in immune response, and those involved in detoxification of xenobiotic molecules from the environment (Thomas 2007), are likely associated with environmental changes during speciation (Lynch and Conery 2000; Zhang 2003). We found a duplication of N6AMT1, a member of a gene family implicated in environmental stress response. N6AMT1 plays a role in detoxification of xenobiotic compounds, more specifically, converting the arsenic metabolite monomethylarsonous acid to the less toxic dimethylarsonic acid (Ren et al. 2011). Chronic arsenic exposure through water, soil and crops, has been monitored in the neotropics, as it represents a severe public health issue, leading to various diseases including cancer (Zhang et al. 2015). This duplication event indicates putative local adaptation of tayra to this xenobiotic compound. 
Furthermore, we also found candidate genes associated with vision and cognition, which may be associated with the tayra's primarily diurnal foraging activity. Tayras supposedly detect prey primarily by smell, as their eyesight has been described as being relatively poor (Galef et al. 1976; Wilson and Mittermeier 2009). This is contradictory to the observed behaviour of caching of unripe but mature stages of both native and non-native fruit by tayras (Soley and Alvarado-Díaz 2011). We detected variation in several genes implicated with lens fiber formation and retina vascularization, including gene expansions of ANKRD13A (Avellino et al. 2013) and RBP2 (D'Ambrosio et al. 2011), along with the inversion of PDC (Pandya et al. 2016), suggesting that tayras might not in fact exhibit poor eyesight. The caching behavior mentioned above (Soley \& Alvaro-Diaz, 2011) indicates remarkable sensory perception, especially olfaction (Presley 2000) and memory and recognition of different stages of fruit development, likely evolved as a response to avoid competition with other species during seasons when fruit becomes ripe. We observed candidate genes related to memory and learning such as SLC38A1 (Qureshi et al. 2019) and CRBN (Higgins et al. 2010) that may have been involved in the evolution of this type of caching behavior. 


\section{Materials and Methods}

\section{Sample, Sequencing, and Genome Assembly}

Whole blood from a captive male tayra was collected by the veterinary staff of the "Wildkatzenzentrum Felidae", Barnim (Germany), during a routine medical checkup. High molecular weight (HMW) genomic DNA extraction was performed using the Qiagen MagAttract HMW DNA Kit, following the manufacturer's protocol. We used 1ng of DNA and the Chromium Genome Reagents Kits Version 2 and the 10X Genomics Chromium Controller instrument with a microfluidic chip for library preparation (10x Genomics). This library was then sequenced on an Illumina NovaSeq 6000 instrument with 300 cycles on an S1 lane at the Max Delbrück Center for Molecular Medicine, Berlin.

Due to uncertainty regarding the optimum number of reads for genome assembly, we generated three de novo genome assemblies using the 10x Genomics Supernova assembler v2.1.1 (Weisenfeld et al. 2017) with default parameters, each with a different read input: 900 million, 1.3 billion and 1.7 billion paired reads, respectively. Based on Supernova assembly metrics (contig, scaffold, and phase block N50 sizes), we chose the assembly generated using 1.3 billion paired reads for further analysis (metrics for the chosen assembly given in SI Table S1).

\section{Assessment of gene completeness}

The assemblies of tayra, wolverine, sable and domestic ferret were assessed for gene completeness with BUSCO v4.1.2 using the mammalian lineage dataset mammalia_odb10 (Simão et al. 2015), which included 9226 single-copy orthologs.

\section{Repetitive Elements}

To accurately identify repeat families, we used RepeatModeler v2 (Flynn et al. 2020) with the option "-LTRstruct" option, followed by Repeatmasker v4.1.2 (Smit 2004) to identify and mask the modeled repeats in the tayra genome assembly. 


\section{Mapping to reference}

For the purpose of demographic history and structural variation analyses, reads from all three Guloninae species were aligned to the domestic ferret (Mustela putorius furo) genome (MusPutFur1.0_HiC; Peng et al. 2014; Dudchenko et al. 2017, 2018) in local mode with Bowtie2 v2.3.5.1 (Langmead and Salzberg 2012). The proportion of reads mapping to the reference genome for all three samples was above $96 \%$. Duplicated reads were removed with Picard Toolkit v2.23 (MarkDuplicates, http://broadinstitute.github.io/picard). Insert size distributions for each sample library (SI Figure S9) were generated with Svtyper v0.7.1 (Chiang et al. 2015). Trimmed tayra reads were downsampled to $\sim 38 \mathrm{x}$ with seqtk $\mathrm{v} 1.3$ (https://github.com//h3/seqtk) prior to mapping to maintain uniformity among libraries and to avoid bias in variant calling.

\section{Pre-processing of sequencing data}

Prior to mapping, linked-read barcodes were trimmed from tayra sequencing reads with proc10xG (filter_Reads.py, https://github.com/ucdavis-bioinformatics/proc10xSC). For the other two species, we used the reads used for generating the respective genome assemblies of Scandinavian wolverine (Gulo gulo, NCBI accession: GCA_900006375.2, PRJEB10674; Ekblom et al. 2018), and sable (Martes zibellina, NCBI accession: GCA_012583365.1, PRJNA495455; Liu et al. 2020). Adapter clipping and quality trimming (Q30, min. length $80 \mathrm{bp}$ ) were performed on all samples with TrimGalore v0.6.4 (Krueger et al. 2021). Additionally, trimmed reads were further used in the nucleotide diversity analysis.

\section{Demographic reconstruction}

Analysis of demographic history was performed with PSMC v0.6.5 (Li and Durbin 2011) using the following parameters (repeated 100 times for bootstrapping): psmc $-\mathrm{N} 25$-t15 $-r 5-b-p$ ' $4+25^{\star} 2+4+66^{\prime}$-o round-\$\{ARRAY_TASK_ID\}.psmc $\$\{$ name $\} . s p l i t . p s m c f a$

Results for each genome were plotted with psmc_plot.pl, and the mutation rate and generation time were set to $1 \mathrm{e}-08$ substitutions per site per generation and 4 years respectively (Cahill et al. 2016; Dobrynin et al. 2015). 


\section{Reference-based scaffolding}

For better visualization of heterozygosity (see below), we generated pseudochromosome assemblies for tayra, wolverine and sable using as reference the chromosome-length domestic ferret (Mustela putorius furo) genome assembly published by DNA Zoo (https://www.dnazoo.org/assemblies/Mustela_putorius_furo). Scaffolding was performed using RaGOO v1.1 (Alonge et al. 2019). The $X$ chromosome in the domestic ferret assembly was identified via whole genome alignment to the domestic cat (Felis catus) Felis_catus_9.0 assembly (Buckley et al. 2020) and ZooFISH data available from the Atlas of Mammalian Chromosomes (Cavagna et al. 2000; O'Brien et al. 2020). Whole genome alignment was performed using LAST v971 (Frith and Kawaguchi 2015).

\section{Alignment to pseudochromosome assemblies and sex verification}

Trimmed reads were aligned to the pseudochromosome assemblies of corresponding species using BWA version 0.7.17 ( $\mathrm{Li}$ and Durbin 2009). Read duplicates were marked using the markdup utility from Samtools version 1.10 (Li 2011). To verify the sex of the tayra, sable and wolverine genomes, two approaches were applied: marker-based and coverage-based. For the marker approach, we used the Y-chromosome-specific SRY gene (sex determining region $Y$ ). The amino acid sequence of the Martes melampus SRY (Genbank ID BAJ05096.1; Yamada and Masuda 2010) was downloaded from the $\mathrm{NCBI}$ protein database and aligned to the pseudochromosome assemblies using Exonerate $\vee$ 2.2.4 (Slater and Birney 2005) in protein2genome mode to identify orthologous sequences. For the second approach, a per-base genome coverage was estimated using Bedtools v2.29 (Quinlan and Hall 2010). Mean and median values for both non-overlapping sliding windows of $1 \mathrm{Mbp}$ and whole genomes were calculated and visualized using scripts from the MACE package (https://github.com/mahajrod/MACE).

\section{Visualization of heterozygosity distribution}

Variant calling followed by quality filtration was performed using the BCFtools pipeline v1.10 (Poplin et al. 2018). Variant filtration was performed in several stages. Low-quality variants were removed using the BCFtools filter with the following thresholds: 'QUAL < $20.0 \| \mid$ (FORMAT/SP > 60.0 || FORMAT/DP < 5.0 || FORMAT/GQ < 20.0)'. Then, variant coverage was investigated. In each sample, positions with coverage lower or higher than $50-250 \%$ of the whole genome median value were removed, and out of the 
remaining positions only those common to all samples were retained. Finally, SNPs with uncalled genotypes in any sample and variants with the same genotypes for all samples were removed. For visualization, heterozygous SNPs were counted in non-overlapping sliding windows of $1 \mathrm{Mbp}$ and counts were scaled to SNPs per kbp. Indels were not included due to the low quality of calls from short reads. SNP density plots were created using the MACE package (https://github.com/mahajrod/MACE).

\section{Phylogenomic data preparation, analysis and dating}

We performed sequence alignments and filtering of highly divergent alignment segments for 6020 coding genomic regions of single-copy orthologs shared across eight species of carnivores, using the software MACSE v2 (Ranwez et al. 2011). These alignments were further trimmed for highly divergent regions, excessive missing data, and substitution model adequacy (further details in Supplementary information). Gene trees were then estimated from acceptable gene regions by first selecting the best substitution model from the GTR $+F+\Gamma+I+R$ family (Kalyaanamoorthy et al. 2017) and calculating approximate likelihood-ratio test (aLRT) branch supports (Anisimova and Gascuel 2006), as implemented in IQ-TREE v2 (Minh et al. 2020).

Species tree estimates were performed using (i) concatenated sequence alignments for maximum likelihood inference using IQ-TREE v2, and (ii) gene trees for inference under the multi-species coalescent using the summary coalescent method in ASTRAL-III (Zhang et al. 2018). The maximum likelihood estimate of the species tree was accompanied by aLRT branch supports, while summary coalescent inference was accompanied by local posterior probabilities (Sayyari and Mirarab 2016). The decisiveness of the data regarding the phylogenetic signals was examined using geneand site-concordance factors, calculated in IQ-TREE v2 (Minh et al. 2020).

Bayesian molecular dating analysis was performed using MCMCtree in PAML v4.8 (Yang 2007), assuming the reconstructed species tree from ASTRAL-III and using the genomic regions with gene trees concordant with the species tree (see Supplementary information for further details). Data were partitioned by codon positions, each modelled under individual GTR $+\Gamma$ substitution models. We used an uncorrelated gamma prior on rates across lineages and a birth-death prior for divergence times. Fossil calibrations included (i) the split between Caniformes and Feliformes using the age of the oldest known fossil of the family Viverravidae (65 - 50Mya; Wang and Tedford 2008; (ii) the split between Canidae and other Caniformes using a fossil of the genus Amphicticeps (32.8 - 30.4Mya; Wang et al. 2005; (iii) the split between Pinnipedia and Musteloidea using fossils of stem musteloids of the genus Mustelictis (32.8 - 23.3Mya; Wang et al. 
2005; Rybczynski et al. 2009; and (iv) the split between the pinnipeds Odobenus and Mirounga, using a fossil of the genus Proneotherium (20.4 - 13.8Mya; Deméré et al. 2003). The posterior distribution was sampled every $1 \times 10^{3}$ MCMC steps over $1 \times 10^{7}$ steps, after a burn-in phase of $1 \times 10^{6}$ steps. We verified convergence to the stationary distribution by comparing the results from two independent runs, and confirming that the effective sample sizes for all parameters were above 1000 using the $R$ package coda (Plummer et al. 2006).

\section{Positive selection on single-copy orthologs}

To investigate genes under positive selection, the CDS sequences corresponding to 1:1 orthologs were aligned for the eight aforementioned carnivoran species. Multiple sequence alignments (MSAs) were constructed with PRANK v120716 (Löytynoja 2014), and 17 MSAs were subsequently filtered out due to the alignments being too short. The CODEML module in the PAML v4.5 package was used to estimate the ratio of non-synonymous to synonymous substitutions, also called $d_{N} / d_{S}$ or $\omega$ (Yang 2007). We applied the one-ratio model to estimate the general selective pressure acting among all species, allowing only a single $d_{N} / d_{S}$ ratio for all branches. A free-ratio model was also used to estimate the $d_{N} / d_{S}$ ratio of each branch. Furthermore, the CODEML branch-site test for positive selection was performed on 6003 ortholog alignments for three separate foreground branches: Eira barbara, Gulo gulo and Martes zibellina (Zhang et al. 2005). Statistical significance was assessed using likelihood ratio tests (LRT) with a conservative $10 \%$ false discovery rate (FDR) criterion (Nielsen et al. 2005). Orthologs with a free-ratio $>2$ in the branch model were considered for further analysis of signatures of positive selection.

To account for differences in genome assembly quality, we evaluated the alignments of selected orthologs based on the transitive consistency score (TCS), an extension to the T-Coffee scoring scheme used to determine the most accurate positions in MSAs (Chang et al. 2014). Additionally, alignments were visually inspected for potential low-scoring MSA portions. Orthologs passing all the filters and inspection were further explored for their putative functional and biological roles using literature sources and online databases, including OrthoDB v10 (Kriventseva et al. 2019), Uniprot (The UniProt Consortium 2017), and NCBI Entrez Gene (Maglott et al. 2011). 


\section{Gene family evolution}

In order to make inferences regarding changes in gene family sizes, we constructed a matrix with counts of complete single-copy, complete duplicated, and missing orthologs identified through the BUSCO genome assembly completeness assessment of all eight carnivoran genomes. Orthologs were retained if they were detected in at least four species (including Felis catus as an outgroup) to obtain meaningful likelihood scores for the global birth and death $(\lambda)$ parameter.

We used CAFE v4.2.1. (Han et al. 2013) to analyse gene gains ('birth') and losses ('death') accounting for phylogenetic history. First, we estimated the error distribution in our dataset, as genome assembly and annotation errors can result in biased estimates of the average rate of change $(\lambda)$, potentially leading to an overestimation of $\lambda$.

Following the error distribution modelling, we ran the CAFE analysis guided by the ultrametric tree estimated earlier, calculating a single $\lambda$ parameter for the whole species tree. The CAFE results were summarized (SI Table 4A) with the python script cafetutorial_report_analysis.py (https://github.com/hahnlab/CAFE). All identified expansions were in the form of gene duplications, with one putative triplication detected in tayra (SI Table S4B). Functional and biological roles associated with gained gene copies for each ortholog in the gulonine species were assessed using literature sources and online databases, including OrthoDB v10 (Kriventseva et al. 2019), Uniprot (The UniProt Consortium 2017), and NCBI Entrez Gene (Maglott et al. 2011).

\section{Structural variation}

We applied an ensemble approach for structural variant (SV) calling, encompassing three SV callers: Manta v1.6.0 (Chen et al. 2016), Whamg v1.7.0 (Kronenberg et al. 2015) and Lumpy v0.2.13 (Layer et al. 2014). SV calls originating from reads mapping in low complexity regions and having poor mapping quality were removed from all three call sets. We retained Manta calls with paired-read (PR) and split-read (SR) support of $P R>=3$ and $S R>=3$, respectively. To reduce the number of false positive calls, the Whamg call set was filtered for potential translocation events, as Whamg flags but does not specifically call translocations. We further removed Whamg calls with $A<10$ (total pieces of evidence supporting the variant: PR, SR). We removed Lumpy calls with a low number of reads supporting the variant (SU) of SU < 10. All SV call sets were filtered based on genotype quality $(G Q \geq 30)$. Whamg and Lumpy SV call sets were genotyped 
with Svtyper v0.7.1 (Chiang et al. 2015) prior to filtering. Only scaffolds assigned to chromosomes were included in further analyses. Survivor v1.0.7 (Jeffares et al. 2017) was used to merge and compare SV call sets within and among samples. The union set of SV calls among the three gulonine species containing species-specific and shared variants (Figure 1.) was annotated. First, Liftoff v1.5.1 (Shumate and Salzberg 2020) was used for preparation of reference genome annotation and Ensembl Variant Effect Predictor v101.0 (McLaren et al. 2016) for identifying variants affecting protein-coding genes.

Functional and biological roles of genes affected by SVs was explored using literature sources and online databases, including OrthoDB v10 (Kriventseva et al. 2019), Uniprot (The UniProt Consortium 2017), and NCBI Entrez Gene (Maglott et al. 2011). Gene ontology analysis was performed with Shiny GO (Ge et al. 2020) with an FDR < 0.05 for each SV type (excluding inversions) overlapping multiple protein-coding genes (> 5 genes).

\section{Data availability}

This whole genome shotgun project has been deposited in GenBank under the accession JAHRIG000000000-JAHRIH000000000. The version described in this paper is version JAHRIG01000000-JAHRIH010000000.

\section{Acknowledgments and funding information}

We thank Dr. Rafael from the Felidae Wildkatzen- und Artenschutzzentrum Barnim for the kind provision of the tayra sample, Michael Hofreiter from the Adaptive Genomics group (University of Potsdam) for facilitating the 10x sequencing.

David Duchêne was funded by a Carlsbergfondet postdoctoral fellowship, grant number CF18-0223.

Sergei Kliver, Andrey Tomarovsky and Azamat Totikov were funded by the Russian Foundation for Basic Research, grant № 20-04-00808. Azamat Totikov and Andrey Tomarovsky were additionally funded by JetBrains Research. 


\section{Competing interests}

Authors declare no competing interests.

\section{References}

1000 Genomes Project Consortium, Adam Auton, Lisa D. Brooks, Richard M. Durbin, Erik P. Garrison, Hyun Min Kang, Jan O. Korbel, et al. 2015. "A Global Reference for Human Genetic Variation." Nature 526 (7571): 68-74.

Abduriyim, S., Y. Nishita, A. V. Abramov, V. A. Solovyev, A. P. Saveljev, P. A. Kosintsev, A. P. Kryukov, et al. 2019. "Variation in Pancreatic Amylase Gene Copy Number among Eurasian Badgers (Carnivora, Mustelidae, Meles ) and Its Relationship to Diet." Journal of Zoology 308 (1): 28-36.

Alonge, Michael, Sebastian Soyk, Srividya Ramakrishnan, Xingang Wang, Sara Goodwin, Fritz J. Sedlazeck, Zachary B. Lippman, and Michael C. Schatz. 2019. "RaGOO: Fast and Accurate Reference-Guided Scaffolding of Draft Genomes." Genome Biology 20 (1): 224.

Al-Qusairi, Lama, Ivana Prokic, Leonela Amoasii, Christine Kretz, Nadia Messaddeq, Jean-Louis Mandel, and Jocelyn Laporte. 2013. "Lack of Myotubularin (MTM1) Leads to Muscle Hypotrophy through Unbalanced Regulation of the Autophagy and Ubiquitin-Proteasome Pathways." FASEB Journal: Official Publication of the Federation of American Societies for Experimental Biology 27 (8): 3384-94.

Amstislavsky, S., and Y. Ternovskaya. 2000. "Reproduction in Mustelids." Animal Reproduction Science 60-61 (July): 571-81.

Anisimova, Maria, and Olivier Gascuel. 2006. "Approximate Likelihood-Ratio Test for Branches: A Fast, Accurate, and Powerful Alternative." Systematic Biology 55 (4): 539-52.

Armstrong, Ellie E., Ryan W. Taylor, Stefan Prost, Peter Blinston, Esther van der Meer, Hillary Madzikanda, Olivia Mufute, et al. 2019. "Cost-Effective Assembly of the African Wild Dog (Lycaon Pictus) Genome Using Linked Reads." GigaScience 8 (2). https://doi.org/10.1093/gigascience/giy124.

Aronsson, Malin, and Jens Persson. 2018. "Female Breeding Dispersal in Wolverines, a Solitary Carnivore with High Territorial Fidelity." European Journal of Wildlife Research 64 (1): 7.

Avellino, Raffaella, Sabrina Carrella, Marinella Pirozzi, Maurizio Risolino, Francesco Giuseppe Salierno, Paola Franco, Patrizia Stoppelli, Pasquale Verde, Sandro Banfi, and Ivan Conte. 2013. "miR-204 Targeting of Ankrd13A Controls Both Mesenchymal Neural Crest and Lens Cell Migration." PloS One 8 (4): e61099.

Avnet, Sofia, Silvia Lemma, Costantino Errani, Luigi Falzetti, Emanuele Panza, Marta 
Columbaro, Cristina Nanni, and Nicola Baldini. 2020. "Benign Albeit Glycolytic: MCT4 Expression and Lactate Release in Giant Cell Tumour of Bone." Bone 134 (May): 115302.

Bähler, Martin, and Allen Rhoads. 2002. "Calmodulin Signaling via the IQ Motif." FEBS Letters 513 (1): 107-13.

Bao, Jianqiang, Jie Zhang, Huili Zheng, Chen Xu, and Wei Yan. 2010. "UBQLN1 Interacts with SPEM1 and Participates in Spermiogenesis." Molecular and Cellular Endocrinology 327 (1-2): 89-97.

Barnett, Ross, Michael V. Westbury, Marcela Sandoval-Velasco, Filipe Garrett Vieira, Sungwon Jeon, Grant Zazula, Michael D. Martin, et al. 2020. "Genomic Adaptations and Evolutionary History of the Extinct Scimitar-Toothed Cat, Homotherium Latidens." Current Biology: CB 30 (24): 5018-25.e5.

Bauer, Anina, Michela De Lucia, Vidhya Jagannathan, Giorgia Mezzalira, Margret L. Casal, Monika M. Welle, and Tosso Leeb. 2017. "A Large Deletion in the NSDHL Gene in Labrador Retrievers with a Congenital Cornification Disorder." G3 7 (9): 3115-21.

Baumann, Peter, Sonja C. Schriever, Stephanie Kullmann, Annemarie Zimprich, Annette Feuchtinger, Oana Amarie, Andreas Peter, et al. 2019. "Dusp8 Affects Hippocampal Size and Behavior in Mice and Humans." Scientific Reports 9 (1): 19483.

Beichman, Annabel C., Klaus-Peter Koepfli, Gang Li, William Murphy, Pasha Dobrynin, Sergei Kilver, M. Tim Tinker, et al. 2019. "Aquatic Adaptation and Depleted Diversity: A Deep Dive into the Genomes of the Sea Otter and Giant Otter." Molecular Biology and Evolution, June. https://doi.org/10.1093/molbev/msz101.

Blottner, Steffen, Jennifer Schön, and Katarina Jewgenow. 2006. "Seasonally Activated Spermatogenesis Is Correlated with Increased Testicular Production of Testosterone and Epidermal Growth Factor in Mink (Mustela Vison)." Theriogenology 66 (6-7): 1593-98.

Blume, Marissa, Fuduki Inoguchi, Taku Sugiyama, Yuji Owada, Noriko Osumi, Yoshinari Aimi, Kosuke Taki, and Yu Katsuyama. 2017. "Dab1 Contributes Differently to the Morphogenesis of the Hippocampal Subdivisions." Development, Growth \& Differentiation 59 (8): 657-73.

Browning, Andrew C., Gustavo S. Figueiredo, Oliver Baylis, Emma Montgomery, Clare Beesley, Elisa Molinari, Francisco C. Figueiredo, and John A. Sayer. 2019. "A Case of Ocular Cystinosis Associated with Two Potentially Severe CTNS Mutations." Ophthalmic Genetics 40 (2): 157-60.

Brown, J. Alison, Dawn M. Eberhardt, F. Neal Schrick, Mary P. Roberts, and James D. Godkin. 2003. "Expression of Retinol-Binding Protein and Cellular Retinol-Binding Protein in the Bovine Ovary." Molecular Reproduction and Development 64 (3): 261-69.

Brown, James H., and Robert C. Lasiewski. 1972. "Metabolism of Weasels: The Cost of Being Long and Thin." Ecology 53 (5): 939-43.

Buckley, Reuben M., Brian W. Davis, Wesley A. Brashear, Fabiana H. G. Farias, Kei Kuroki, Tina Graves, Ladeana W. Hillier, et al. 2020. "A New Domestic Cat Genome Assembly Based on Long Sequence Reads Empowers Feline Genomic Medicine 
and Identifies a Novel Gene for Dwarfism." PLoS Genetics 16 (10): e1008926.

Cabral, Wayne A., Masaki Ishikawa, Matthias Garten, Elena N. Makareeva, Brandi M. Sargent, Maryann Weis, Aileen M. Barnes, et al. 2016. "Absence of the ER Cation Channel TMEM38B/TRIC-B Disrupts Intracellular Calcium Homeostasis and Dysregulates Collagen Synthesis in Recessive Osteogenesis Imperfecta." PLoS Genetics 12 (7): e1006156.

Cahill, James A., André E. R. Soares, Richard E. Green, and Beth Shapiro. 2016. "Inferring Species Divergence Times Using Pairwise Sequential Markovian Coalescent Modelling and Low-Coverage Genomic Data." Philosophical Transactions of the Royal Society of London. Series B, Biological Sciences 371 (1699). https://doi.org/10.1098/rstb.2015.0138.

Carmo, Sonia Do, David Fournier, Catherine Mounier, and Eric Rassart. 2009. "Human Apolipoprotein D Overexpression in Transgenic Mice Induces Insulin Resistance and Alters Lipid Metabolism." American Journal of Physiology-Endocrinology and Metabolism 296 (4): E802-11.

Catanach, Andrew, Ross Crowhurst, Cecilia Deng, Charles David, Louis Bernatchez, and Maren Wellenreuther. 2019. "The Genomic Pool of Standing Structural Variation Outnumbers Single Nucleotide Polymorphism by Threefold in the Marine Teleost Chrysophrys Auratus." Molecular Ecology 28 (6): 1210-23.

Cavagna, P., A. Menotti, and R. Stanyon. 2000. "Genomic Homology of the Domestic Ferret with Cats and Humans." Mammalian Genome: Official Journal of the International Mammalian Genome Society 11 (10): 866-70.

Ceballos, Francisco C., Peter K. Joshi, David W. Clark, Michèle Ramsay, and James F. Wilson. 2018. "Runs of Homozygosity: Windows into Population History and Trait Architecture." Nature Reviews. Genetics 19 (4): 220-34.

Chang, Jia-Ming, Paolo Di Tommaso, and Cedric Notredame. 2014. "TCS: A New Multiple Sequence Alignment Reliability Measure to Estimate Alignment Accuracy and Improve Phylogenetic Tree Reconstruction." Molecular Biology and Evolution 31 (6): 1625-37.

Chen, Di, Joyce M. Teng, Paula E. North, Philip E. Lapinski, and Philip D. King. 2019. "RASA1-Dependent Cellular Export of Collagen IV Controls Blood and Lymphatic Vascular Development." The Journal of Clinical Investigation 129 (9): 3545-61.

Chen, Xiaoyu, Ole Schulz-Trieglaff, Richard Shaw, Bret Barnes, Felix Schlesinger, Morten Källberg, Anthony J. Cox, Semyon Kruglyak, and Christopher T. Saunders. 2016. "Manta: Rapid Detection of Structural Variants and Indels for Germline and Cancer Sequencing Applications." Bioinformatics 32 (8): 1220-22.

Chiang, Colby, Ryan M. Layer, Gregory G. Faust, Michael R. Lindberg, David B. Rose, Erik P. Garrison, Gabor T. Marth, Aaron R. Quinlan, and Ira M. Hall. 2015. "SpeedSeq: Ultra-Fast Personal Genome Analysis and Interpretation." Nature Methods 12 (10): 966-68.

Chiang, Colby, Alexandra J. Scott, Joe R. Davis, Emily K. Tsang, Xin Li, Yungil Kim, Tarik Hadzic, et al. 2017. "The Impact of Structural Variation on Human Gene Expression." Nature Genetics 49 (5): 692-99.

Coan, P. M., O. R. Vaughan, Y. Sekita, S. L. Finn, G. J. Burton, M. Constancia, and A. L. Fowden. 2010. "Adaptations in Placental Phenotype Support Fetal Growth during 
Undernutrition of Pregnant Mice." The Journal of Physiology 588 (Pt 3): 527-38.

Copeland, J. P., and T. E. Kucera. 1997. "Wolverine (Gulo Gulo)." The Wildlife Society California North.

https://www.originalwisdom.com/wp-content/uploads/bsk-pdf-manager/2019/03/Har ris-et-al_1997_Mesocarnivores-of-Northern-California.pdf\#page=33.

Crocco, Paolina, Adolfo Saiardi, Miranda S. Wilson, Raffaele Maletta, Amalia C. Bruni, Giuseppe Passarino, and Giuseppina Rose. 2016. "Contribution of Polymorphic Variation of Inositol Hexakisphosphate Kinase 3 (IP6K3) Gene Promoter to the Susceptibility to Late Onset Alzheimer's Disease." Biochimica et Biophysica Acta 1862 (9): 1766-73.

Crow, Yanick J., Diana S. Chase, Johanna Lowenstein Schmidt, Marcin Szynkiewicz, Gabriella M. A. Forte, Hannah L. Gornall, Anthony Oojageer, et al. 2015. "Characterization of Human Disease Phenotypes Associated with Mutations in TREX1, RNASEH2A, RNASEH2B, RNASEH2C, SAMHD1, ADAR, and IFIH1." American Journal of Medical Genetics. Part A 167A (2): 296-312.

D’Ambrosio, Diana N., Robin D. Clugston, and William S. Blaner. 2011. "Vitamin A Metabolism: An Update." Nutrients 3 (1): 63-103.

Davydova, Erna, Angela Y. Y. Ho, Jedrzej Malecki, Anders Moen, Jorrit M. Enserink, Magnus E. Jakobsson, Christoph Loenarz, and Pål Ø. Falnes. 2014. "Identification and Characterization of a Novel Evolutionarily Conserved Lysine-Specific Methyltransferase Targeting Eukaryotic Translation Elongation Factor 2 (eEF2)." The Journal of Biological Chemistry 289 (44): 30499-510.

De la Chapelle, A., J. Schröder, and M. Pernu. 1972. "Isochromosome for the Short Arm of X, a Human 46, XXpi Syndrome." Annals of Human Genetics 36 (1): 79-87.

Del Dotto, Valentina, Mario Fogazza, Francesco Musiani, Alessandra Maresca, Serena J. Aleo, Leonardo Caporali, Chiara La Morgia, et al. 2018. "Deciphering OPA1 Mutations Pathogenicity by Combined Analysis of Human, Mouse and Yeast Cell Models." Biochimica et Biophysica Acta, Molecular Basis of Disease 1864 (10): 3496-3514.

Deméré, Thomas A., Annalisa Berta, and Peter J. Adam. 2003. "Chapter 3." Bulletin of the American Museum of Natural History 2003 (279): 32-76.

Denker, H. W. 2000. "Structural Dynamics and Function of Early Embryonic Coats." Cells, Tissues, Organs 166 (2): 180-207.

DeSelm, Carl J., Brian C. Miller, Wei Zou, Wandy L. Beatty, Eline van Meel, Yoshifumi Takahata, Judith Klumperman, Sharon A. Tooze, Steven L. Teitelbaum, and Herbert W. Virgin. 2011. "Autophagy Proteins Regulate the Secretory Component of Osteoclastic Bone Resorption." Developmental Cell 21 (5): 966-74.

Dobrynin, Pavel, Shiping Liu, Gaik Tamazian, Zijun Xiong, Andrey A. Yurchenko, Ksenia Krasheninnikova, Sergey Kliver, et al. 2015. "Genomic Legacy of the African Cheetah, Acinonyx Jubatus." Genome Biology 16 (December): 277.

Dong, Fenglan, Kyosuke Shinohara, Yanick Botilde, Ryo Nabeshima, Yasuko Asai, Akemi Fukumoto, Toshiaki Hasegawa, et al. 2014. "Pih1d3 Is Required for Cytoplasmic Preassembly of Axonemal Dynein in Mouse Sperm." The Journal of Cell Biology 204 (2): 203-13.

Dreiza, Catherine M., Padmini Komalavilas, Elizabeth J. Furnish, Charles R. Flynn, 
Michael R. Sheller, Christopher C. Smoke, Luciana B. Lopes, and Colleen M. Brophy. 2010. "The Small Heat Shock Protein, HSPB6, in Muscle Function and Disease." Cell Stress \& Chaperones 15 (1): 1-11.

Dubost, Gérard, and Olivier Henry. 2017. "Seasonal Reproduction in Neotropical Rainforest Mammals." Zoological Studies 56 (January): e2.

Dudchenko, Olga, Sanjit S. Batra, Arina D. Omer, Sarah K. Nyquist, Marie Hoeger, Neva C. Durand, Muhammad S. Shamim, et al. 2017. "De Novo Assembly of the Aedes Aegypti Genome Using Hi-C Yields Chromosome-Length Scaffolds." Science 356 (6333): 92-95.

Dudchenko, Olga, Muhammad S. Shamim, Sanjit S. Batra, Neva C. Durand, Nathaniel T. Musial, Ragib Mostofa, Melanie Pham, et al. 2018. "The Juicebox Assembly Tools Module Facilitates de Novo Assembly of Mammalian Genomes with Chromosome-Length Scaffolds for under \$1000." https://doi.org/10.1101/254797.

Ekblom, Robert, Birte Brechlin, Jens Persson, Linnéa Smeds, Malin Johansson, Jessica

Magnusson, Øystein Flagstad, and Hans Ellegren. 2018. "Genome Sequencing and Conservation Genomics in the Scandinavian Wolverine Population." Conservation Biology: The Journal of the Society for Conservation Biology 32 (6): 1301-12.

Ellerman, Diego A., Jimin Pei, Surabhi Gupta, William J. Snell, Diana Myles, and Paul Primakoff. 2009. "Izumo Is Part of a Multiprotein Family Whose Members Form Large Complexes on Mammalian Sperm." Molecular Reproduction and Development 76 (12): 1188-99.

Etherington, Graham J., Darren Heavens, David Baker, Ashleigh Lister, Rose McNelly, Gonzalo Garcia, Bernardo Clavijo, lain Macaulay, Wilfried Haerty, and Federica Di Palma. 2020. "Sequencing Smart: De Novo Sequencing and Assembly Approaches for a Non-Model Mammal." GigaScience 9 (5). https://doi.org/10.1093/gigascience/giaa045.

Fahien, Leonard A., and Michael J. Macdonald. 2011. "The Complex Mechanism of Glutamate Dehydrogenase in Insulin Secretion." Diabetes 60 (10): 2450-54.

Fenelon, Jane C., Geoffrey Shaw, Stephen R. Frankenberg, Bruce D. Murphy, and Marilyn B. Renfree. 2017. "Embryo Arrest and Reactivation: Potential Candidates Controlling Embryonic Diapause in the Tammar Wallaby and Mink†." Biology of Reproduction 96 (4): 877-94.

Feng, Wei, and Mingjie Zhang. 2009. "Organization and Dynamics of PDZ-Domain-Related Supramodules in the Postsynaptic Density." Nature Reviews. Neuroscience 10 (2): 87-99.

Filatov, Dmitry A., and Dave T. Gerrard. 2003. "High Mutation Rates in Human and Ape Pseudoautosomal Genes." Gene 317 (1-2): 67-77.

Flynn, Jullien M., Robert Hubley, Clément Goubert, Jeb Rosen, Andrew G. Clark, Cédric Feschotte, and Arian F. Smit. 2020. "RepeatModeler2 for Automated Genomic Discovery of Transposable Element Families." Proceedings of the National Academy of Sciences of the United States of America 117 (17): 9451-57.

Forde, Niamh, Constantine A. Simintiras, Roger Sturmey, Solomon Mamo, Alan K. Kelly, Thomas E. Spencer, Fuller W. Bazer, and Pat Lonergan. 2014. "Amino Acids in the Uterine Luminal Fluid Reflects the Temporal Changes in Transporter 
Expression in the Endometrium and Conceptus during Early Pregnancy in Cattle." PloS One 9 (6): e100010.

Frith, Martin C., and Risa Kawaguchi. 2015. "Split-Alignment of Genomes Finds Orthologies More Accurately." Genome Biology 16 (May): 106.

Galef, Bennett G., Russell A. Mittermeier, and Robert C. Bailey. 1976. "Predation by the Tayra (Eira Barbara)." Journal of Mammalogy 57 (4): 760-61.

Geisinger, Adriana, Manfred Alsheimer, Andrea Baier, Ricardo Benavente, and Rodolfo Wettstein. 2005. "The Mammalian Gene Pecanex 1 Is Differentially Expressed during Spermatogenesis." Biochimica et Biophysica Acta 1728 (1-2): 34-43.

Gerits, Nancy, Werner Van Belle, and Ugo Moens. 2007. "Transgenic Mice Expressing Constitutive Active MAPKAPK5 Display Gender-Dependent Differences in Exploration and Activity." Behavioral and Brain Functions: BBF 3 (November): 58.

Ge, Steven Xijin, Dongmin Jung, and Runan Yao. 2020. "ShinyGO: A Graphical Gene-Set Enrichment Tool for Animals and Plants." Bioinformatics 36 (8): 2628-29.

Gòdia, Marta, Joaquim Casellas, Aurora Ruiz-Herrera, Joan E. Rodríguez-Gil, Anna Castelló, Armand Sánchez, and Alex Clop. 2020. "Whole Genome Sequencing Identifies Allelic Ratio Distortion in Sperm Involving Genes Related to Spermatogenesis in a Swine Model." DNA Research: An International Journal for Rapid Publication of Reports on Genes and Genomes 27 (5). https://doi.org/10.1093/dnares/dsaa019.

Goldman, B. D. 1999. "The Circadian Timing System and Reproduction in Mammals." Steroids 64 (9): 679-85.

Gosejacob, Dominic, Philipp S. Jäger, Katharina Vom Dorp, Martin Frejno, Anne C. Carstensen, Monika Köhnke, Joachim Degen, Peter Dörmann, and Michael Hoch. 2016. "Ceramide Synthase 5 Is Essential to Maintain C16:0-Ceramide Pools and Contributes to the Development of Diet-Induced Obesity." The Journal of Biological Chemistry 291 (13): 6989-7003.

Grayson, Phil. 2015. "Izumo1 and Juno: The Evolutionary Origins and Coevolution of Essential Sperm-Egg Binding Partners." Royal Society Open Science 2 (12): 150296.

Grayson, Phil, and Alberto Civetta. 2012. "Positive Selection and the Evolution of Izumo Genes in Mammals." International Journal of Evolutionary Biology 2012 (August): 958164.

Guo, H. X., W. Cun, L. D. Liu, S. Z. Dong, L. C. Wang, C. H. Dong, and Q. H. Li. 2006. "Protein Encoded by HSV-1 Stimulation-Related Gene 1 (HSRG1) Interacts with and Inhibits SV40 Large T Antigen." Cell Proliferation 39 (6): 507-18.

Gurevich, Alexey, Vladislav Saveliev, Nikolay Vyahhi, and Glenn Tesler. 2013. "QUAST: Quality Assessment Tool for Genome Assemblies." Bioinformatics 29 (8): 1072-75.

Gu, Sumin, Hywel Llewelyn Roderick, Patricia Camacho, and Jean X. Jiang. 2001. "Characterization of an N-System Amino Acid Transporter Expressed in Retina and Its Involvement in Glutamine Transport ${ }^{*}$." The Journal of Biological Chemistry 276 (26): 24137-44.

Han, Mira V., Gregg W. C. Thomas, Jose Lugo-Martinez, and Matthew W. Hahn. 2013. "Estimating Gene Gain and Loss Rates in the Presence of Error in Genome 
Assembly and Annotation Using CAFE 3." Molecular Biology and Evolution 30 (8): 1987-97.

Han, Xuelei, Tengfei Jiang, Lei Yu, Cuiping Zeng, Bin Fan, and Bang Liu. 2012. "Molecular Characterization of the Porcine MTPAP Gene Associated with Meat Quality Traits: Chromosome Localization, Expression Distribution, and Transcriptional Regulation." Molecular and Cellular Biochemistry 364 (1-2): 173-80.

Harney, J. P., T. L. Ott, R. D. Geisert, and F. W. Bazer. 1993. "Retinol-Binding Protein Gene Expression in Cyclic and Pregnant Endometrium of Pigs, Sheep, and Cattle." Biology of Reproduction 49 (5): 1066-73.

Hecker, Nikolai, Virag Sharma, and Michael Hiller. 2019. "Convergent Gene Losses Illuminate Metabolic and Physiological Changes in Herbivores and Carnivores." Proceedings of the National Academy of Sciences of the United States of America 116 (8): 3036-41.

Heldstab, Sandra A., Dennis W. H. Müller, Sereina M. Graber, Laurie Bingaman Lackey, Eberhard Rensch, Jean-Michel Hatt, Philipp Zerbe, and Marcus Clauss. 2018. "Geographical Origin, Delayed Implantation, and Induced Ovulation Explain Reproductive Seasonality in the Carnivora." Journal of Biological Rhythms 33 (4): 402-19.

Higgins, Joseph J., Adit L. Tal, Xiaowei Sun, Stefanie C. R. Hauck, Jin Hao, Barry E. Kosofosky, and Anjali M. Rajadhyaksha. 2010. "Temporal and Spatial Mouse Brain Expression of Cereblon, an Ionic Channel Regulator Involved in Human Intelligence." Journal of Neurogenetics 24 (1): 18-26.

Hill, Martin, Antonín Pařízek, David Cibula, Radmila Kancheva, Jan Evangelista Jirásek, Marie Jirkovská, Marta Velíková, et al. 2010. "Steroid Metabolome in Fetal and Maternal Body Fluids in Human Late Pregnancy." The Journal of Steroid Biochemistry and Molecular Biology 122 (4): 114-32.

Hill, M., A. Pařízek, R. Kancheva, and J. E. Jirásek. 2011. "Reduced Progesterone Metabolites in Human Late Pregnancy." Physiological Research / Academia Scientiarum Bohemoslovaca 60 (2): 225-41.

Hnia, Karim, Helene Tronchère, Kinga K. Tomczak, Leonela Amoasii, Patrick Schultz, Alan H. Beggs, Bernard Payrastre, Jean Louis Mandel, and Jocelyn Laporte. 2011. "Myotubularin Controls Desmin Intermediate Filament Architecture and Mitochondrial Dynamics in Human and Mouse Skeletal Muscle." The Journal of Clinical Investigation 121 (1): 70-85.

Horibata, Yasuhiro, Orly Elpeleg, Ayelet Eran, Yoshio Hirabayashi, David Savitzki, Galit Tal, Hanna Mandel, and Hiroyuki Sugimoto. 2018. "EPT1 (selenoprotein I) Is Critical for the Neural Development and Maintenance of Plasmalogen in Humans." Journal of Lipid Research 59 (6): 1015-26.

Hron, Tomáš, Daniel Elleder, and Robert J. Gifford. 2019. "Deltaretroviruses Have Circulated since at Least the Paleogene and Infected a Broad Range of Mammalian Species." Retrovirology 16 (1): 33.

Im, Sin-Hyeog, Soo-Hyun Kim, Tania Azam, Natarajan Venkatesh, Charles A. Dinarello, Sara Fuchs, and Miriam C. Souroujon. 2002. "Rat Interleukin-18 Binding Protein: Cloning, Expression, and Characterization." Journal of Interferon \& Cytokine Research: The Official Journal of the International Society for Interferon and 
Cytokine Research 22 (3): 321-28.

Inman, Robert M., Audrey J. Magoun, Jens Persson, and Jenny Mattisson. 2012. "The Wolverine's Niche: Linking Reproductive Chronology, Caching, Competition, and Climate." Journal of Mammalogy 93 (3): 634-44.

Inoue, Kana, Norikazu Maeda, Takuya Mori, Ryohei Sekimoto, Yu Tsushima, Keisuke Matsuda, Masaya Yamaoka, et al. 2014. "Possible Involvement of Opa-Interacting Protein 5 in Adipose Proliferation and Obesity." PloS One 9 (2): e87661.

Ishizawa, Toshihiro, Yusuke Nozaki, Takuya Ueda, and Nono Takeuchi. 2008. "The Human Mitochondrial Translation Release Factor HMRF1L Is Methylated in the GGQ Motif by the Methyltransferase HMPrmC." Biochemical and Biophysical Research Communications 373 (1): 99-103.

Jeffares, Daniel C., Clemency Jolly, Mimoza Hoti, Doug Speed, Liam Shaw, Charalampos Rallis, Francois Balloux, Christophe Dessimoz, Jürg Bähler, and Fritz J. Sedlazeck. 2017. "Transient Structural Variations Have Strong Effects on Quantitative Traits and Reproductive Isolation in Fission Yeast." Nature Communications 8 (January): 14061.

Jewgenow, Katarina, Frank Goeritz, Katrin Neubauer, Joerns Fickel, and Sergej V. Naidenko. 2006. "Characterization of Reproductive Activity in Captive Male Eurasian Lynx (Lynx Lynx)." European Journal of Wildlife Research 52 (1): 34-38.

Johnson, Richard J., and Peter Andrews. 2010. "Fructose, Uricase, and the Back-to-Africa Hypothesis." Evolutionary Anthropology 19 (6): 250-57.

Johnson, Richard J., Yuri Y. Sautin, William J. Oliver, Carlos Roncal, Wei Mu, L. Gabriela Sanchez-Lozada, Bernardo Rodriguez-Iturbe, Takahiko Nakagawa, and Steven A. Benner. 2009. "Lessons from Comparative Physiology: Could Uric Acid Represent a Physiologic Alarm Signal Gone Awry in Western Society?" Journal of Comparative Physiology. B, Biochemical, Systemic, and Environmental Physiology 179 (1): 67-76.

Johnson, R. J., P. Stenvinkel, P. Andrews, L. G. Sánchez-Lozada, T. Nakagawa, E. Gaucher, A. Andres-Hernando, et al. 2020. "Fructose Metabolism as a Common Evolutionary Pathway of Survival Associated with Climate Change, Food Shortage and Droughts." Journal of Internal Medicine 287 (3): 252-62.

Kalyaanamoorthy, Subha, Bui Quang Minh, Thomas K. F. Wong, Arndt von Haeseler, and Lars S. Jermiin. 2017. "ModelFinder: Fast Model Selection for Accurate Phylogenetic Estimates." Nature Methods 14 (6): 587-89.

Kang, Cong Bao, Ye Hong, Sirano Dhe-Paganon, and Ho Sup Yoon. 2008. "FKBP Family Proteins: Immunophilins with Versatile Biological Functions." Neuro-Signals 16 (4): 318-25.

Kashtanov, S. N., G. R. Svischeva, S. L. Pishchulina, O. E. Lazebny, I. G. Meshchersky, L. V. Simakin, and V. V. Rozhnov. 2015. "Geographical Structure of the Sable (Martes Zibellina L.) Gene Pool on the Basis of Microsatellite Loci Analysis." Russian Journal of Genetics 51 (1): 69-79.

Kazmierczak, Marcin, Suzan L. Harris, Piotr Kazmierczak, Prahar Shah, Valentin Starovoytov, Kevin K. Ohlemiller, and Martin Schwander. 2015. "Progressive Hearing Loss in Mice Carrying a Mutation in Usp53." The Journal of Neuroscience: The Official Journal of the Society for Neuroscience 35 (47): 15582-98. 
Kim, Bo-Mi, Yoon Jin Lee, Jeong-Hoon Kim, Jin-Hyoung Kim, Seunghyun Kang, Euna Jo, Seung Jae Lee, Jun Hyuck Lee, Young Min Chi, and Hyun Park. 2020. "The Genome Assembly and Annotation of the Southern Elephant Seal Mirounga Leonina." Genes 11 (2). https://doi.org/10.3390/genes11020160.

Kim, Jihye, Kei-Ichiro Ishiguro, Aya Nambu, Bungo Akiyoshi, Shihori Yokobayashi, Ayano Kagami, Tadashi Ishiguro, et al. 2015. "Meikin Is a Conserved Regulator of Meiosis-I-Specific Kinetochore Function." Nature 517 (7535): 466-71.

Kim, Jun Hyun, Channabasavaiah Basavaraju Gurumurthy, Hamid Band, and Vimla Band. 2010. "Biochemical Characterization of Human Ecdysoneless Reveals a Role in Transcriptional Regulation." Biological Chemistry 391 (1): 9-19.

Kitchener, Andrew C., Carlo Meloro, and Terrie M. Williams. 2018. "Form and Function of the Musteloids." In Biology and Conservation of Musteloids. Oxford University Press.

Koepfli, Klaus-Peter, Kerry A. Deere, Graham J. Slater, Colleen Begg, Keith Begg, Lon Grassman, Mauro Lucherini, Geraldine Veron, and Robert K. Wayne. 2008. "Multigene Phylogeny of the Mustelidae: Resolving Relationships, Tempo and Biogeographic History of a Mammalian Adaptive Radiation." BMC Biology 6 (February): 10.

Kondo, Shu, and Ryu Ueda. 2013. "Highly Improved Gene Targeting by Germline-Specific Cas9 Expression in Drosophila." Genetics 195 (3): 715-21.

Kosiol, Carolin, Tomás Vinar, Rute R. da Fonseca, Melissa J. Hubisz, Carlos D. Bustamante, Rasmus Nielsen, and Adam Siepel. 2008. "Patterns of Positive Selection in Six Mammalian Genomes." PLoS Genetics 4 (8): e1000144.

Koskela, Anni, Ilpo Kojola, Jouni Aspi, and Marja Hyvärinen. 2013. "The Diet of Breeding Female Wolverines (Gulo Gulo) in Two Areas of Finland." Acta Theriologica 58 (2): 199-204.

Kosugi, Shunichi, Yukihide Momozawa, Xiaoxi Liu, Chikashi Terao, Michiaki Kubo, and Yoichiro Kamatani. 2019. "Comprehensive Evaluation of Structural Variation Detection Algorithms for Whole Genome Sequencing." Genome Biology 20 (1): 117.

Kratzer, James T., Miguel A. Lanaspa, Michael N. Murphy, Christina Cicerchi, Christina L. Graves, Peter A. Tipton, Eric A. Ortlund, Richard J. Johnson, and Eric A. Gaucher. 2014. "Evolutionary History and Metabolic Insights of Ancient Mammalian Uricases." Proceedings of the National Academy of Sciences of the United States of America 111 (10): 3763-68.

Krebs, John, Eric Lofroth, Jeffrey Copeland, Vivian Banci, Dorothy Cooley, Howard Golden, Audrey Magoun, Robert Mulders, and Brad Shults. 2004. "Synthesis of Survival Rates and Causes of Mortality in North American Wolverines." The Journal of Wildlife Management 68 (3): 493-502.

Kriventseva, Evgenia V., Dmitry Kuznetsov, Fredrik Tegenfeldt, Mosè Manni, Renata Dias, Felipe A. Simão, and Evgeny M. Zdobnov. 2019. "OrthoDB v10: Sampling the Diversity of Animal, Plant, Fungal, Protist, Bacterial and Viral Genomes for Evolutionary and Functional Annotations of Orthologs." Nucleic Acids Research 47 (D1): D807-11.

Kronenberg, Zev N., Edward J. Osborne, Kelsey R. Cone, Brett J. Kennedy, Eric T. 
Domyan, Michael D. Shapiro, Nels C. Elde, and Mark Yandell. 2015. "Wham: Identifying Structural Variants of Biological Consequence." PLoS Computational Biology 11 (12): e1004572.

Krueger, Felix, Frankie James, Phil Ewels, Ebrahim Afyounian, and Benjamin Schuster-Boeckler. 2021. FelixKrueger/TrimGalore: v0.6.7 - DOI via Zenodo. https://doi.org/10.5281/zenodo.5127899.

Kuriakose, Teneema, and Thirumala-Devi Kanneganti. 2018. "ZBP1: Innate Sensor

Regulating Cell Death and Inflammation." Trends in Immunology 39 (2): 123-34.

Langmead, Ben, and Steven L. Salzberg. 2012. "Fast Gapped-Read Alignment with Bowtie 2." Nature Methods 9 (4): 357-59.

Larivière, Serge, and Steven H. Ferguson. 2003. "Evolution of Induced Ovulation in North American Carnivores." Journal of Mammalogy 84 (3): 937-47.

Larsen, Knud, Jamal Momeni, Leila Farajzadeh, and Henrik Callesen. 2017. "Splice Variants of Porcine PPHLN1 Encoding Periphilin-1." Gene Reports 7 (June): 176-83.

Law, Chris J., Graham J. Slater, and Rita S. Mehta. 2018. "Lineage Diversity and Size Disparity in Musteloidea: Testing Patterns of Adaptive Radiation Using Molecular and Fossil-Based Methods." Systematic Biology 67 (1): 127-44.

Layer, Ryan M., Colby Chiang, Aaron R. Quinlan, and Ira M. Hall. 2014. "LUMPY: A Probabilistic Framework for Structural Variant Discovery." Genome Biology 15 (6): R84.

Lee, Jong-Eun, Hyun-Ah Oh, Haengseok Song, Jin Hyun Jun, Cheong-Rae Roh, Huirong Xie, S. K. Dey, and Hyunjung Jade Lim. 2011. "Autophagy Regulates Embryonic Survival during Delayed Implantation." Endocrinology 152 (5): 2067-75.

Lennox, Alanda R., and Allen E. Goodship. 2008. "Polar Bears (Ursus Maritimus), the Most Evolutionary Advanced Hibernators, Avoid Significant Bone Loss during Hibernation." Comparative Biochemistry and Physiology. Part A, Molecular \& Integrative Physiology 149 (2): 203-8.

Lewin, Harris A., Jennifer A. Marshall Graves, Oliver A. Ryder, Alexander S. Graphodatsky, and Stephen J. O'Brien. 2019. "Precision Nomenclature for the New Genomics." GigaScience 8 (8). https://doi.org/10.1093/gigascience/giz086.

Liberles, Stephen D. 2015. "Trace Amine-Associated Receptors: Ligands, Neural Circuits, and Behaviors." Current Opinion in Neurobiology 34 (October): 1-7.

$\mathrm{Li}$, Bo, Mieczyslaw Wolsan, Dan Wu, Wei Zhang, Yanchun Xu, and Zhaohui Zeng. 2014. "Mitochondrial Genomes Reveal the Pattern and Timing of Marten (Martes), Wolverine (Gulo), and Fisher (Pekania) Diversification." Molecular Phylogenetics and Evolution 80 (November): 156-64.

$\mathrm{Li}$, Heng. 2011. "A Statistical Framework for SNP Calling, Mutation Discovery, Association Mapping and Population Genetical Parameter Estimation from Sequencing Data." Bioinformatics 27 (21): 2987-93.

$\mathrm{Li}$, Heng, and Richard Durbin. 2009. "Fast and Accurate Short Read Alignment with Burrows-Wheeler Transform." Bioinformatics 25 (14): 1754-60.

- 2011. "Inference of Human Population History from Individual Whole-Genome Sequences." Nature 475 (7357): 493-96.

Lim, Hyunjung J., and Haengseok Song. 2014. "Evolving Tales of Autophagy in Early 
Reproductive Events." The International Journal of Developmental Biology 58 (2-4): 183-87.

Li, Qian, Wayne J. Korzan, David M. Ferrero, Rui B. Chang, Dheeraj S. Roy, Mélanie Buchi, Jamie K. Lemon, et al. 2013. "Synchronous Evolution of an Odor Biosynthesis Pathway and Behavioral Response." Current Biology: CB 23 (1): 11-20.

Liu, Guangshuai, Chao Zhao, Dongming Xu, Huanxin Zhang, Vladimir Monakhov, Shuai Shang, Xiaodong Gao, et al. 2020. "First Draft Genome of the Sable, Martes Zibellina." Genome Biology and Evolution 12 (3): 59-65.

Liu, Lu, Shuaiyi Chen, Miao Yu, Chenxu Ge, Mengmeng Ren, Boya Liu, Xin Yang, et al. 2020. "Deacetylation of HSD17B10 by SIRT3 Regulates Cell Growth and Cell Resistance under Oxidative and Starvation Stresses." Cell Death \& Disease 11 (7): 563.

Li, Xuemei, Yan Ye, Baoli Wang, and Shujun Zhao. 2021. "miR-140-5p Aggravates Insulin Resistance via Directly Targeting GYS1 and PPP1CC in Insulin-Resistant HepG2 Cells." Diabetes, Metabolic Syndrome and Obesity: Targets and Therapy 14 (June): 2515-24.

Lopes, Flavia L., Joëlle Desmarais, Nicolas Y. Gevry, Sandra Ledoux, and Bruce D. Murphy. 2003. "Expression of Vascular Endothelial Growth Factor Isoforms and Receptors Flt-1 and KDR during the Peri-Implantation Period in the Mink, Mustela Vison." Biology of Reproduction 68 (5): 1926-33.

Lopes-Marques, Monica, Catarina Serrano, Ana R. Cardoso, Renato Salazar, Susana Seixas, António Amorim, Luisa Azevedo, and Maria J. Prata. 2020. "GBA3: A Polymorphic Pseudogene in Humans That Experienced Repeated Gene Loss during Mammalian Evolution." Scientific Reports 10 (1): 11565.

Löytynoja, Ari. 2014. "Phylogeny-Aware Alignment with PRANK." Methods in Molecular Biology 1079: 155-70.

Luczkowska, Karolina, Caroline Stekelenburg, Frédérique Sloan-Béna, Emmanuelle Ranza, Giacomo Gastaldi, Valérie Schwitzgebel, and Pierre Maechler. 2020. "Hyperinsulinism Associated with GLUD1 Mutation: Allosteric Regulation and Functional Characterization of p.G446V Glutamate Dehydrogenase." Human Genomics 14 (1): 9.

Lynch, M., and J. S. Conery. 2000. "The Evolutionary Fate and Consequences of Duplicate Genes." Science.

Maglott, Donna, Jim Ostell, Kim D. Pruitt, and Tatiana Tatusova. 2011. "Entrez Gene: Gene-Centered Information at NCBI." Nucleic Acids Research 39 (Database issue): D52-57.

Marín, Ignacio. 2014. "The Ubiquilin Gene Family: Evolutionary Patterns and Functional Insights." BMC Evolutionary Biology 14 (March): 63.

Martell, K. J., A. F. Seasholtz, S. P. Kwak, K. K. Clemens, and J. E. Dixon. 1995. "hVH-5: A Protein Tyrosine Phosphatase Abundant in Brain That Inactivates Mitogen-Activated Protein Kinase." Journal of Neurochemistry 65 (4): 1823-33.

Martinez, Bridget, and Rudy M. Ortiz. 2017. "Thyroid Hormone Regulation and Insulin Resistance: Insights From Animals Naturally Adapted to Fasting." Physiology, February. https://doi.org/10.1152/physiol.00018.2016. 
Masure, S., M. Cik, E. Hoefnagel, C. A. Nosrat, I. Van der Linden, R. Scott, P. Van Gompel, et al. 2000. "Mammalian GFRalpha -4, a Divergent Member of the GFRalpha Family of Coreceptors for Glial Cell Line-Derived Neurotrophic Factor Family Ligands, Is a Receptor for the Neurotrophic Factor Persephin." The Journal of Biological Chemistry 275 (50): 39427-34.

Matsubara, Takuma, Shoichiro Kokabu, Chihiro Nakatomi, Masayuki Kinbara, Toshihiro Maeda, Mitsuhiro Yoshizawa, Hisataka Yasuda, Teruko Takano-Yamamoto, Roland Baron, and Eijiro Jimi. 2018. "The Actin-Binding Protein PPP1r18 Regulates Maturation, Actin Organization, and Bone Resorption Activity of Osteoclasts." Molecular and Cellular Biology 38 (4). https://doi.org/10.1128/MCB.00425-17.

Mattisson, Jenny, Geir Rune Rauset, John Odden, Henrik Andrén, John D. C. Linnell, and Jens Persson. 2017. "Predation or Scavenging? Prey Body Condition Influences Decision-Making in a Facultative Predator, the Wolverine." The Bulletin of the Ecological Society of America 98 (1): 40-46.

McDaniel, Patrick, and Xuemei Wu. 2009. "Identification of Oocyte-Selective NLRP Genes in Rhesus Macaque Monkeys (Macaca Mulatta)." Molecular Reproduction and Development 76 (2): 151-59.

McGee-Lawrence, Meghan, Patricia Buckendahl, Caren Carpenter, Kim Henriksen, Michael Vaughan, and Seth Donahue. 2015. "Suppressed Bone Remodeling in Black Bears Conserves Energy and Bone Mass during Hibernation." The Journal of Experimental Biology 218 (Pt 13): 2067-74.

McLaren, William, Laurent Gil, Sarah E. Hunt, Harpreet Singh Riat, Graham R. S. Ritchie, Anja Thormann, Paul Flicek, and Fiona Cunningham. 2016. "The Ensembl Variant Effect Predictor." Genome Biology 17 (1): 122.

McNutt, J. W., R. Groom, and R. Woodroffe. 2019. "Ambient Temperature Provides an Adaptive Explanation for Seasonal Reproduction in a Tropical Mammal." Journal of Zoology 309 (3): 153-60.

Mead, Rodney A. 1981. "Delayed Implantation in Mustelids, with Special Emphasis on the Spotted Skunk." Journal of Reproduction and Fertility. Supplement 29: 11-24.

- 1989. "The Physiology and Evolution of Delayed Implantation in Carnivores." In Carnivore Behavior, Ecology, and Evolution, edited by John L. Gittleman, 437-64. Boston, MA: Springer US.

Mérot, Claire, Rebekah A. Oomen, Anna Tigano, and Maren Wellenreuther. 2020. "A Roadmap for Understanding the Evolutionary Significance of Structural Genomic Variation." Trends in Ecology \& Evolution, April. https://doi.org/10.1016/j.tree.2020.03.002.

Metzger, Julia, Matthias Karwath, Raul Tonda, Sergi Beltran, Lídia Águeda, Marta Gut, Ivo Glynne Gut, and Ottmar Distl. 2015. "Runs of Homozygosity Reveal Signatures of Positive Selection for Reproduction Traits in Breed and Non-Breed Horses." BMC Genomics 16 (October): 764.

Minh, Bui Quang, Matthew W. Hahn, and Robert Lanfear. 2020. "New Methods to Calculate Concordance Factors for Phylogenomic Datasets." Molecular Biology and Evolution 37 (9): 2727-33.

Minh, Bui Quang, Heiko A. Schmidt, Olga Chernomor, Dominik Schrempf, Michael D. Woodhams, Arndt von Haeseler, and Robert Lanfear. 2020. "IQ-TREE 2: New 
Models and Efficient Methods for Phylogenetic Inference in the Genomic Era." Molecular Biology and Evolution 37 (5): 1530-34.

Miranda, Inês, Iwona Giska, Liliana Farelo, João Pimenta, Marketa Zimova, Jarosław Bryk, Love Dalén, L. Scott Mills, Karol Zub, and José Melo-Ferreira. 2021. "Museomics Dissects the Genetic Basis for Adaptive Seasonal Colouration in the Least Weasel." Molecular Biology and Evolution, June. https://doi.org/10.1093/molbev/msab177.

Mironova, Ekaterina, and Clarke F. Millette. 2008. "Expression of the Diaphanous-Related Formin Proteins mDia1 and mDia2 in the Rat Testis." Developmental Dynamics: An Official Publication of the American Association of Anatomists 237 (8): 2170-76.

Mizuarai, Shinji, Satomi Miki, Hiromitsu Araki, Kazuhiko Takahashi, and Hidehito Kotani. 2005. "Identification of Dicarboxylate Carrier Slc25a10 as Malate Transporter in de Novo Fatty Acid Synthesis." The Journal of Biological Chemistry 280 (37): 32434-41.

Monakhov, Vladimir G. 2011. "Martes Zibellina (Carnivora: Mustelidae)." Mammalian Species 43 (876): 75-86.

Montanez, Eloi, Sara A. Wickström, Johannes Altstätter, Haiyan Chu, and Reinhard Fässler. 2009. "Alpha-Parvin Controls Vascular Mural Cell Recruitment to Vessel Wall by Regulating RhoA/ROCK Signalling." The EMBO Journal 28 (20): 3132-44.

Montaseri, Azadeh, Claudia Giampietri, Michela Rossi, Anna Riccioli, Andrea Del Fattore, and Antonio Filippini. 2020. "The Role of Autophagy in Osteoclast Differentiation and Bone Resorption Function." Biomolecules 10 (10). https://doi.org/10.3390/biom10101398.

Murphy, B. D. 2012. "Embryonic Diapause: Advances in Understanding the Enigma of Seasonal Delayed Implantation." Reproduction in Domestic Animals = Zuchthygiene 47 Suppl 6 (December): 121-24.

Murphy, B. D., and J. C. Fenelon. 2020. "Models of Embryonic Diapause in Carnivora." Bioscientifica Proceedings, June. https://doi.org/10.1530/biosciprocs.10.006.

Mustonen, Anne-Mari, Reijo Käkelä, Anne Käkelä, Teija Pyykönen, Jari Aho, and Petteri Nieminen. 2007. "Lipid Metabolism in the Adipose Tissues of a Carnivore, the Raccoon Dog, during Prolonged Fasting." Experimental Biology and Medicine 232 (1): 58-69.

Mustonen, Anne-Mari, Matti Puukka, Seppo Saarela, Tommi Paakkonen, Jari Aho, and Petteri Nieminen. 2006. "Adaptations to Fasting in a Terrestrial Mustelid, the Sable (Martes Zibellina)." Comparative Biochemistry and Physiology. Part A, Molecular \& Integrative Physiology 144 (4): 444-50.

Nakamura, Satoki, Tomoaki Kahyo, Hong Tao, Kiyoshi Shibata, Nobuya Kurabe, Hidetaka Yamada, Kazuya Shinmura, Kazunori Ohnishi, and Haruhiko Sugimura. 2015. "Novel Roles for LIX1L in Promoting Cancer Cell Proliferation through ROS1-Mediated LIX1L Phosphorylation." Scientific Reports 5 (August): 13474.

Nelson, Lenny, Susan Anderson, Alan L. Archibald, Susan Rhind, Zen H. Lu, Alison Condie, Neal McIntyre, et al. 2008. "An Animal Model to Evaluate the Function and Regulation of the Adaptively Evolving Stress Protein SEP53 in Oesophageal Bile Damage Responses." Cell Stress \& Chaperones 13 (3): 375-85. 
Nielsen, Rasmus, Carlos Bustamante, Andrew G. Clark, Stephen Glanowski, Timothy B. Sackton, Melissa J. Hubisz, Adi Fledel-Alon, et al. 2005. "A Scan for Positively Selected Genes in the Genomes of Humans and Chimpanzees." PLoS Biology 3 (6): e170.

Nieminen, Petteri, Reijo Käkelä, Teija Pyykönen, and Anne-Mari Mustonen. 2006. "Selective Fatty Acid Mobilization in the American Mink (Mustela Vison) during Food Deprivation." Comparative Biochemistry and Physiology. Part B, Biochemistry \& Molecular Biology 145 (1): 81-93.

Nieminen, Petteri, and Anne-Mari Mustonen. 2007. "Uniform Fatty Acid Mobilization from Anatomically Distinct Fat Depots in the Sable (Martes Zibellina)." Lipids 42 (7): 659-69.

Nomura, Yuichiro, Daniel Roston, Eric J. Montemayor, Qiang Cui, and Samuel E. Butcher. 2018. "Structural and Mechanistic Basis for Preferential Deadenylation of U6 snRNA by Usb1." Nucleic Acids Research 46 (21): 11488-501.

O'Brien, Stephen J., Alexander S. Graphodatsky, and Polina L. Perelman. 2020. Atlas of Mammalian Chromosomes. John Wiley \& Sons.

Oda, Masako, Yoko Satta, Osamu Takenaka, and Naoyuki Takahata. 2002. "Loss of Urate Oxidase Activity in Hominoids and Its Evolutionary Implications." Molecular Biology and Evolution 19 (5): 640-53.

Okamoto, Takumi, Kazunori Imaizumi, and Masayuki Kaneko. 2020. "The Role of Tissue-Specific Ubiquitin Ligases, RNF183, RNF186, RNF182 and RNF152, in Disease and Biological Function." International Journal of Molecular Sciences 21 (11). https://doi.org/10.3390/ijms21113921.

Olson, M. V. 1999. "When Less Is More: Gene Loss as an Engine of Evolutionary Change." American Journal of Human Genetics 64 (1): 18-23.

Pandya, Dipal Y., Divyesh N. Kelawala, Namrata V. Patel, Tejas M. Shah, Anand B. Patel, Nidhi R. Parmar, Bhaskar Reddy, Deepak B. Patil, and Chaitanya G. Joshi. 2016. "Identification of Genetic Variants in PDC, RHO, PDE6A and PDE6B in Dogs with Progressive Retinal Atrophy." Current Science 111 (10): 1640-48.

Papin, Catherine, Christel Rouget, Thierry Lorca, Anna Castro, and Elisabeth Mandart. 2004. "XCdh1 Is Involved in Progesterone-Induced Oocyte Maturation." Developmental Biology 272 (1): 66-75.

Paschen, S. A., U. Rothbauer, K. Káldi, M. F. Bauer, W. Neupert, and M. Brunner. 2000. "The Role of the TIM8-13 Complex in the Import of Tim23 into Mitochondria." The EMBO Journal 19 (23): 6392-6400.

Pasitschniak-Arts, Maria, and Serge Larivière. 1995. "Gulo Gulo." Mammalian Species, no. 499 (June): 1-10.

Peng, Changjun, Lili Niu, Jiabo Deng, Jianqiu Yu, Xueyan Zhang, Chuang Zhou, Jinchuan Xing, and Jing Li. 2018. "Can-SINE Dynamics in the Giant Panda and Three Other Caniformia Genomes." Mobile DNA 9 (November): 32.

Peng, Xinxia, Jessica Alföldi, Kevin Gori, Amie J. Eisfeld, Scott R. Tyler, Jennifer Tisoncik-Go, David Brawand, et al. 2014. "The Draft Genome Sequence of the Ferret (Mustela Putorius Furo) Facilitates Study of Human Respiratory Disease." Nature Biotechnology 32 (12): 1250-55.

Persson, Jens. 2005. "Female Wolverine (Gulo Gulo) Reproduction: Reproductive Costs 
and Winter Food Availability." Canadian Journal of Zoology 83 (11): 1453-59.

Persson, Sara, Britt-Marie Bäcklin, Hans Kindahl, Björn Brunström, and Ulf Magnusson. 2011. "Influence of Age, Nutritional Status and Season on the Reproductive System in Wild Male Mink (Neovison Vison)." European Journal of Wildlife Research 57 (5): 1057-63.

Picollo, Alessandra, and Michael Pusch. 2005. "Chloride/proton Antiporter Activity of Mammalian CLC Proteins CIC-4 and CIC-5." Nature 436 (7049): 420-23.

Pierre, Karin, Annabelle Parent, Pierre-Yves Jayet, Andrew P. Halestrap, Urs Scherrer, and Luc Pellerin. 2007. "Enhanced Expression of Three Monocarboxylate Transporter Isoforms in the Brain of Obese Mice." The Journal of Physiology 583 (Pt 2): 469-86.

Pintus, Eliana, José Luis Ros-Santaella, and José Julián Garde. 2015. "Beyond Testis Size: Links between Spermatogenesis and Sperm Traits in a Seasonal Breeding Mammal." PloS One 10 (10): e0139240.

Plummer, Martyn, Nicky Best, Kate Cowles, and Karen Vines. 2006. "CODA: Convergence Diagnosis and Output Analysis for MCMC." R News 6 (1): 7-11.

Poglayen-Neuwall, Ivo, Barbara S. Durrant, Marda L. Swansen, Robert C. Williams, and Roy A. Barnes. 1989. "Estrous Cycle of the tayra,Eira Barbara." Zoo Biology 8 (2): 171-77.

Pontremoli, Chiara, Diego Forni, Uberto Pozzoli, Mario Clerici, Rachele Cagliani, and Manuela Sironi. 2021. "Kinetochore Proteins and Microtubule-Destabilizing Factors Are Fast Evolving in Eutherian Mammals." Molecular Ecology 30 (6): 1505-15.

Poon, Connie E., Laura Lecce, Margot L. Day, and Christopher R. Murphy. 2014. "Mucin 15 Is Lost but Mucin 13 Remains in Uterine Luminal Epithelial Cells and the Blastocyst at the Time of Implantation in the Rat." Reproduction, Fertility, and Development 26 (3): 421-31.

Poplin, Ryan, Valentin Ruano-Rubio, Mark A. DePristo, Tim J. Fennell, Mauricio O. Carneiro, Geraldine A. Van der Auwera, David E. Kling, et al. 2018. "Scaling Accurate Genetic Variant Discovery to Tens of Thousands of Samples." bioRxiv. https://doi.org/10.1101/201178.

Porubsky, David, Ashley D. Sanders, Wolfram Höps, Pinghsun Hsieh, Arvis Sulovari, Ruiyang Li, Ludovica Mercuri, et al. 2020. "Recurrent Inversion Toggling and Great Ape Genome Evolution." Nature Genetics 52 (8): 849-58.

Premzl, Marko. 2016. "Comparative Genomic Analysis of Eutherian Tumor Necrosis Factor Ligand Genes." Immunogenetics 68 (2): 125-32.

Presley, Steven J. 2000. "Eira Barbara." Mammalian Species 2000 (636): 1-6.

Proulx, Gilbert, and Keith B. Aubry. 2017. "The Martes Complex: A Monophyletic Clade That Shares Many Life-History Traits and Conservation Challenges." 2017. https://www.fs.fed.us/pnw/pubs/journals/pnw_2017_aubry001.pdf.

Quinlan, Aaron R., and Ira M. Hall. 2010. "BEDTools: A Flexible Suite of Utilities for Comparing Genomic Features." Bioinformatics 26 (6): 841-42.

Qureshi, Tayyaba, Christina Sørensen, Paul Berghuis, Vidar Jensen, Marton B. Dobszay, Tamás Farkas, Knut Tomas Dalen, et al. 2019. "The Glutamine Transporter Slc38a1 Regulates GABAergic Neurotransmission and Synaptic Plasticity." Cerebral Cortex 29 (12): 5166-79. 
Ranwez, Vincent, Sébastien Harispe, Frédéric Delsuc, and Emmanuel J. P. Douzery. 2011. "MACSE: Multiple Alignment of Coding SEquences Accounting for Frameshifts and Stop Codons." PloS One 6 (9): e22594.

Rauniyar, Khushbu, Sawan Kumar Jha, and Michael Jeltsch. 2018. "Biology of Vascular Endothelial Growth Factor C in the Morphogenesis of Lymphatic Vessels." Frontiers in Bioengineering and Biotechnology 6 (February): 7.

Reis, Alexandra, Heng-Yu Chang, Mark Levasseur, and Keith T. Jones. 2006. "APCcdh1 Activity in Mouse Oocytes Prevents Entry into the First Meiotic Division." Nature Cell Biology 8 (5): 539-40.

Renfree, Marilyn B., and Jane C. Fenelon. 2017. "The Enigma of Embryonic Diapause." Development 144 (18): 3199-3210.

Ren, Xuefeng, Maria Aleshin, William J. Jo, Russel Dills, David A. Kalman, Christopher D. Vulpe, Martyn T. Smith, and Luoping Zhang. 2011. "Involvement of N-6 Adenine-Specific DNA Methyltransferase 1 (N6AMT1) in Arsenic Biomethylation and Its Role in Arsenic-Induced Toxicity." Environmental Health Perspectives 119 (6): 771-77.

Reynolds, April, Huanyu Qiao, Ye Yang, Jefferson K. Chen, Neil Jackson, Kajal Biswas, J. Kim Holloway, et al. 2013. "RNF212 Is a Dosage-Sensitive Regulator of Crossing-over during Mammalian Meiosis." Nature Genetics 45 (3): 269-78.

Rinker, David C., Natalya K. Specian, Shu Zhao, and John G. Gibbons. 2019. "Polar Bear Evolution Is Marked by Rapid Changes in Gene Copy Number in Response to Dietary Shift." Proceedings of the National Academy of Sciences of the United States of America 116 (27): 13446-51.

Rismanchi, Neggy, Cynthia Soderblom, Julia Stadler, Peng-Peng Zhu, and Craig Blackstone. 2008. "Atlastin GTPases Are Required for Golgi Apparatus and ER Morphogenesis." Human Molecular Genetics 17 (11): 1591-1604.

Robitaille, Jean-François, Liane Villano, Thomas S. Jung, Helen P. Slama, and Michelle P. Oakley. 2012. "Fat Dynamics and Development of Body Condition Indices for Harvested Populations of Wolverine Gulo Gulo." Wildlife Biology 18 (1): 35-45.

Rybczynski, Natalia, Mary R. Dawson, and Richard H. Tedford. 2009. "A Semi-Aquatic Arctic Mammalian Carnivore from the Miocene Epoch and Origin of Pinnipedia." Nature 458 (7241): 1021-24.

Salleron, Lisa, Giovanni Magistrelli, Camille Mary, Nicolas Fischer, Amos Bairoch, and Lydie Lane. 2014. "DERA Is the Human Deoxyribose Phosphate Aldolase and Is Involved in Stress Response." Biochimica et Biophysica Acta 1843 (12): 2913-25.

Sato, Jun J., Mieczyslaw Wolsan, Francisco J. Prevosti, Guillermo D’Elía, Colleen Begg, Keith Begg, Tetsuji Hosoda, Kevin L. Campbell, and Hitoshi Suzuki. 2012. "Evolutionary and Biogeographic History of Weasel-like Carnivorans (Musteloidea)." Molecular Phylogenetics and Evolution 63 (3): 745-57.

Sayyari, Erfan, and Siavash Mirarab. 2016. "Fast Coalescent-Based Computation of Local Branch Support from Quartet Frequencies." Molecular Biology and Evolution 33 (7): 1654-68.

Schulte-Hostedde, Albrecht I., Bertram Zinner, John S. Millar, and Graham J. Hickling. 2005. "Restitution of Mass-size Residuals: Validating Body Condition Indices." Ecology 86 (1): 155-63. 
Schweitzer, Lawrence D., William C. Comb, Liron Bar-Peled, and David M. Sabatini. 2015. "Disruption of the Rag-Ragulator Complex by c17orf59 Inhibits mTORC1." Cell Reports 12 (9): 1445-55.

Serão, N. V. L., R. Veroneze, A. M. F. Ribeiro, L. L. Verardo, J. Braccini Neto, E. Gasparino, C. F. Campos, P. S. Lopes, and S. E. F. Guimarães. 2011. "Candidate Gene Expression and Intramuscular Fat Content in Pigs." Journal of Animal Breeding and Genetics = Zeitschrift Fur Tierzuchtung Und Zuchtungsbiologie 128 (1): 28-34.

Shaheen, Ranad, Nan Jiang, Fatema Alzahrani, Nour Ewida, Tarfa Al-Sheddi, Eman Alobeid, Damir Musaev, et al. 2019. "Bi-Allelic Mutations in FAM149B1 Cause Abnormal Primary Cilium and a Range of Ciliopathy Phenotypes in Humans." American Journal of Human Genetics 104 (4): 731-37.

Shang, Jun, Tian Xia, Qiang-Qiang Han, Xiaolu Zhao, Ming-Ming Hu, Hong-Bing Shu, and Lin Guo. 2018. "Quantitative Proteomics Identified TTC4 as a TBK1 Interactor and a Positive Regulator of SeV-Induced Innate Immunity." Proteomics 18 (2). https://doi.org/10.1002/pmic.201700403.

Sharma, Virag, Nikolai Hecker, Juliana G. Roscito, Leo Foerster, Bjoern E. Langer, and Michael Hiller. 2018. "A Genomics Approach Reveals Insights into the Importance of Gene Losses for Mammalian Adaptations." Nature Communications 9 (1): 1215.

Shi, Guangsen, Lijuan Xing, Zhiwei Liu, Zhipeng Qu, Xi Wu, Zhen Dong, Xiaohan Wang, et al. 2013. "Dual Roles of FBXL3 in the Mammalian Circadian Feedback Loops Are Important for Period Determination and Robustness of the Clock." Proceedings of the National Academy of Sciences of the United States of America 110 (12): 4750-55.

Shi, Yu-Qiang, Yin-Chuan Li, Xiao-Qian Hu, Tao Liu, Shang-Ying Liao, Jian Guo, Lin Huang, et al. 2009. "Male Germ Cell-Specific Protein Trs4 Binds to Multiple Proteins." Biochemical and Biophysical Research Communications 388 (3): 583-88.

Shumate, Alaina, and Steven L. Salzberg. 2020. "Liftoff: An Accurate Gene Annotation Mapping Tool." https://doi.org/10.1101/2020.06.24.169680.

Shyu, Ming-Kwang, Mei-Chun Lin, Jin-Chung Shih, Chien-Nan Lee, John Huang, Chian-Hui Liao, I-Fang Huang, Hsiao-Yu Chen, Min-Chuan Huang, and Fon-Jou Hsieh. 2007. "Mucin 15 Is Expressed in Human Placenta and Suppresses Invasion of Trophoblast-like Cells in Vitro." Human Reproduction 22 (10): 2723-32.

Siepka, Sandra M., Seung-Hee Yoo, Junghea Park, Weimin Song, Vivek Kumar, Yinin $\mathrm{Hu}$, Choogon Lee, and Joseph S. Takahashi. 2007. "Circadian Mutant Overtime Reveals F-Box Protein FBXL3 Regulation of Cryptochrome and Period Gene Expression." Cell 129 (5): 1011-23.

Simão, Felipe A., Robert M. Waterhouse, Panagiotis loannidis, Evgenia V. Kriventseva, and Evgeny M. Zdobnov. 2015. "BUSCO: Assessing Genome Assembly and Annotation Completeness with Single-Copy Orthologs." Bioinformatics 31 (19): 3210-12.

Singh, Bhairab N., Wuming Gong, Satyabrata Das, Joshua W. M. Theisen, Javier E. Sierra-Pagan, Demetris Yannopoulos, Erik Skie, Pruthvi Shah, Mary G. Garry, and Daniel J. Garry. 2019. "Etv2 Transcriptionally Regulates Yes1 and Promotes Cell 
Proliferation during Embryogenesis." Scientific Reports 9 (1): 9736.

Slater, Guy St C., and Ewan Birney. 2005. "Automated Generation of Heuristics for Biological Sequence Comparison." BMC Bioinformatics 6 (February): 31.

Smit, A. F. A. 2004. "Repeat-Masker Open-3.0." Http://www. Repeatmasker. Org. https://ci.nii.ac.jp/naid/10029514778/.

Soley, Fernando G., and Isaías Alvarado-Díaz. 2011. "Prospective Thinking in a Mustelid? Eira Barbara (Carnivora) Cache Unripe Fruits to Consume Them Once Ripened." Die Naturwissenschaften 98 (8): 693-98.

Sonna, Larry A., Jun Fujita, Stephen L. Gaffin, and Craig M. Lilly. 2002. "Invited Review: Effects of Heat and Cold Stress on Mammalian Gene Expression." Journal of Applied Physiology 92 (4): 1725-42.

Sun-Wada, G. H., H. Murakami, H. Nakai, Y. Wada, and M. Futai. 2001. "Mouse Atp6f, the Gene Encoding the 23-kDa Proteolipid of Vacuolar Proton Translocating ATPase." Gene 274 (1-2): 93-99.

Svishcheva, G. R., and S. N. Kashtanov. 2011. "Reproductive Strategy of the Sable (Martes Zibellina Linnaeus, 1758): An Analysis of Litter Size Inheritance in Farm-Raised Populations." Russian Journal of Genetics: Applied Research 1 (3): 221-25.

Tavori, Hagai, Shirya Rashid, and Sergio Fazio. 2015. "On the Function and Homeostasis of PCSK9: Reciprocal Interaction with LDLR and Additional Lipid Effects." Atherosclerosis 238 (2): 264-70.

The UniProt Consortium. 2017. "UniProt: The Universal Protein Knowledgebase." Nucleic Acids Research 45 (D1): D158-69.

Thomas, James H. 2007. "Rapid Birth-Death Evolution Specific to Xenobiotic Cytochrome P450 Genes in Vertebrates." PLoS Genetics 3 (5): e67.

Tigano, Anna, Jocelyn P. Colella, and Matthew D. MacManes. 2020. "Comparative and Population Genomics Approaches Reveal the Basis of Adaptation to Deserts in a Small Rodent." Molecular Ecology 29 (7): 1300-1314.

Totikov, Azamat, Andrey Tomarovsky, Dmitry Prokopov, Aliya Yakupova, Tatiana Bulyonkova, Lorena Derezanin, Dmitry Rasskazov, et al. 2021. "Chromosome-Level Genome Assemblies Expand Capabilities of Genomics for Conservation Biology." Genes 12 (9): 1336.

Tsantoulas, Christoforos, Franziska Denk, Massimo Signore, Mohammed A. Nassar, Kensuke Futai, and Stephen B. McMahon. 2018. "Mice Lacking Kcns1 in Peripheral Neurons Show Increased Basal and Neuropathic Pain Sensitivity." Pain 159 (8): 1641-51.

Valente, Pierluigi, Alessandra Romei, Manuela Fadda, Bruno Sterlini, Davide Lonardoni, Nicola Forte, Floriana Fruscione, et al. 2019. "Constitutive Inactivation of the PRRT2 Gene Alters Short-Term Synaptic Plasticity and Promotes Network Hyperexcitability in Hippocampal Neurons." Cerebral Cortex 29 (5): 2010-33.

Viragh, P. A. de, M. Huber, and D. Hohl. 1994. "Involucrin mRNA Is More Abundant in Human Hair Follicles than in Normal Epidermis." The Journal of Investigative Dermatology 103 (6): 815-19.

Viscarra, Jose A., Ruben Rodriguez, Jose Pablo Vazquez-Medina, Andrew Lee, Michael S. Tift, Stephen K. Tavoni, Daniel E. Crocker, and Rudy M. Ortiz. 2013. "Insulin and 
GLP-1 Infusions Demonstrate the Onset of Adipose-Specific Insulin Resistance in a Large Fasting Mammal: Potential Glucogenic Role for GLP-1." Physiological Reports 1 (2): e00023.

Wagener, Asja, Steffen Blottner, Frank Göritz, Wolf Jürgen Streich, and Jörns Fickel. 2003. "Differential Changes in Expression of a and B FGF, IGF-1 and -2, and TGF-Alpha during Seasonal Growth and Involution of Roe Deer Testis." Growth Factors 21 (2): 95-102.

Wang, Xiaoming, Malcolm C. McKenna, and Demberelyin Dashzeveg. 2005. "Amphicticeps and Amphicynodon (Arctoidea, Carnivora) from Hsanda Gol Formation, Central Mongolia and Phylogeny of Basal Arctoids with Comments on Zoogeography." American Museum Novitates 2005 (3483): 1-60.

Wang, Xiaoming, and Richard H. Tedford. 2008. Dogs: Their Fossil Relatives and Evolutionary History. Columbia University Press.

Weischenfeldt, Joachim, Orsolya Symmons, François Spitz, and Jan O. Korbel. 2013. "Phenotypic Impact of Genomic Structural Variation: Insights from and for Human Disease." Nature Reviews. Genetics 14 (2): 125-38.

Weisenfeld, Neil I., Vijay Kumar, Preyas Shah, Deanna M. Church, and David B. Jaffe. 2017. "Direct Determination of Diploid Genome Sequences." Genome Research 27 (5): 757-67.

Weissensteiner, Matthias H., Ignas Bunikis, Ana Catalán, Kees-Jan Francoijs, Ulrich Knief, Wieland Heim, Valentina Peona, et al. 2020. "Discovery and Population Genomics of Structural Variation in a Songbird Genus." Nature Communications 11 (1): 3403.

Wellenreuther, Maren, and Louis Bernatchez. 2018. "Eco-Evolutionary Genomics of Chromosomal Inversions." Trends in Ecology \& Evolution 33 (6): 427-40.

Wikström, Anne M., and Leo Dunkel. 2011. "Klinefelter Syndrome." Best Practice \& Research. Clinical Endocrinology \& Metabolism 25 (2): 239-50.

Wilcox, Andrew, Kostas D. Katsanakis, Farheen Bheda, and T. S. Pillay. 2004. "Asb6, an Adipocyte-Specific Ankyrin and SOCS Box Protein, Interacts with APS to Enable Recruitment of Elongins B and C to the Insulin Receptor Signaling Complex." The Journal of Biological Chemistry 279 (37): 38881-88.

Wild, Thomas, Magda Budzowska, Susanne Hellmuth, Susana Eibes, Gopal Karemore, Marin Barisic, Olaf Stemmann, and Chunaram Choudhary. 2018. "Deletion of APC7 or APC16 Allows Proliferation of Human Cells without the Spindle Assembly Checkpoint." Cell Reports 25 (9): 2317-28.e5.

Wiley, S. R., L. Cassiano, T. Lofton, T. Davis-Smith, J. A. Winkles, V. Lindner, H. Liu, T. O. Daniel, C. A. Smith, and W. C. Fanslow. 2001. "A Novel TNF Receptor Family Member Binds TWEAK and Is Implicated in Angiogenesis." Immunity 15 (5): 837-46.

Wilson, Don E., and Russel A. Mittermeier, eds. 2009. "Family Mustelidae." In Handbook of the Mammals of the World, Vol. 1 Carnivores, 1:627-37. Lynx Editions, Barcelona.

Wu, Xunwei, Fabio Quondamatteo, Tine Lefever, Aleksandra Czuchra, Hannelore Meyer, Anna Chrostek, Ralf Paus, Lutz Langbein, and Cord Brakebusch. 2006. "Cdc42 Controls Progenitor Cell Differentiation and Beta-Catenin Turnover in Skin." 
Genes \& Development 20 (5): 571-85.

Wu, Zhihong, Ties Latendorf, Ulf Meyer-Hoffert, and Jens-M Schröder. 2011. "Identification of Trichohyalin-like 1, an s100 Fused-Type Protein Selectively Expressed in Hair Follicles." The Journal of Investigative Dermatology 131 (8): 1761-63.

Xiao, Qianjun, Xiao-Lin Wu, Jennifer J. Michal, Jerry J. Reeves, Jan R. Busboom, Gary H. Thorgaard, and Zhihua Jiang. 2006. "A Novel Nuclear-Encoded Mitochondrial poly(A) Polymerase PAPD1 Is a Potential Candidate Gene for the Extreme Obesity Related Phenotypes in Mammals." International Journal of Biological Sciences 2 (4): 171-78.

Xie, Li, Wen-Xin Qin, Xiang-Huo He, Hui-Qun Shu, Gen-Fu Yao, Da-Fang Wan, and Jian-Ren Gu. 2004. "Differential Gene Expression in Human Hepatocellular Carcinoma Hep3B Cells Induced by Apoptosis-Related Gene BNIPL-2." World Journal of Gastroenterology: WJG 10 (9): 1286-91.

Xie, Xian-Kuan, Zheng-Kuan Xu, Kan Xu, and Yu-Xiang Xiao. 2020. "DUSP19 Mediates Spinal Cord Injury-Induced Apoptosis and Inflammation in Mouse Primary Microglia Cells via the NF-kB Signaling Pathway." Neurological Research 42 (1): 31-38.

Xu, Zhijie, Lifang Yang, San Xu, Zhibao Zhang, and Ya Cao. 2015. "The Receptor Proteins: Pivotal Roles in Selective Autophagy." Acta Biochimica et Biophysica Sinica 47 (8): 571-80.

Yamada, Chiemi, and Ryuichi Masuda. 2010. "Molecular Phylogeny and Evolution of Sex-Chromosomal Genes and SINE Sequences in the Family Mustelidae." Mammal Study 35 (1): 17-30.

Yamakoshi, Takako, Teruhiko Makino, Mati Ur Rehman, Yoko Yoshihisa, Michiya Sugimori, and Tadamichi Shimizu. 2013. "Trichohyalin-like 1 Protein, a Member of Fused S100 Proteins, Is Expressed in Normal and Pathologic Human Skin." Biochemical and Biophysical Research Communications 432 (1): 66-72.

Yang, Ziheng. 2007. "PAML 4: Phylogenetic Analysis by Maximum Likelihood." Molecular Biology and Evolution 24 (8): 1586-91.

Ye, Wenfeng, Yi Zhou, Bin Xu, Dawei Zhu, Xiaohui Rui, Ming Xu, Lijuan Shi, Dachuan Zhang, and Jingting Jiang. 2019. "CD247 Expression Is Associated with Differentiation and Classification in Ovarian Cancer." Medicine 98 (51): e18407.

Ye, Zhengmao, and Jenny Pan-Yun Ting. 2008. "NLR, the Nucleotide-Binding Domain Leucine-Rich Repeat Containing Gene Family." Current Opinion in Immunology 20 (1): 3-9.

Yockey, Laura J., and Akiko Iwasaki. 2018. "Interferons and Proinflammatory Cytokines in Pregnancy and Fetal Development." Immunity 49 (3): 397-412.

Yu, Yu-Hsiang, Yi-Cheng Chang, Tseng-Hsiung Su, Jiun-Yi Nong, Chao-Chin Li, and Lee-Ming Chuang. 2013. "Prostaglandin Reductase-3 Negatively Modulates Adipogenesis through Regulation of PPARy Activity." Journal of Lipid Research 54 (9): 2391-99.

Zhang, Chao, Maryam Rabiee, Erfan Sayyari, and Siavash Mirarab. 2018. "ASTRAL-III: Polynomial Time Species Tree Reconstruction from Partially Resolved Gene Trees." BMC Bioinformatics 19 (Suppl 6): 153.

Zhang, Cuiping, and Ying Liang. 2018. "Latexin and Hematopoiesis." Current Opinion in 
Hematology 25 (4): 266-72.

Zhang, Hao, Yichen Ge, Ping He, Xushen Chen, Abreu Carina, Yulan Qiu, Diana S. Aga, and Xuefeng Ren. 2015. "Interactive Effects of N6AMT1 and As3MT in Arsenic Biomethylation." Toxicological Sciences: An Official Journal of the Society of Toxicology 146 (2): 354-62.

Zhang, Jianzhi. 2003. "Evolution by Gene Duplication: An Update." Trends in Ecology \& Evolution 18 (6): 292-98.

Zhang, Jianzhi, Rasmus Nielsen, and Ziheng Yang. 2005. "Evaluation of an Improved Branch-Site Likelihood Method for Detecting Positive Selection at the Molecular Level." Molecular Biology and Evolution 22 (12): 2472-79.

Zhao, Chao, Guangshuai Liu, Shuai Shang, Qinguo Wei, Lei Zhang, Tian Xia, Xiufeng Yang, Guolei Sun, and Honghai Zhang. 2019. "Adaptive Evolution of the ACSL Gene Family in Carnivora." Genetica 147 (2): 141-48.

Zhao, Huijie, Lei Zhu, Yunlu Zhu, Jingli Cao, Shanshan Li, Qiongping Huang, Tao Xu, Xiao Huang, Xiumin Yan, and Xueliang Zhu. 2013. "The Cep63 Paralogue Deup1 Enables Massive de Novo Centriole Biogenesis for Vertebrate Multiciliogenesis." Nature Cell Biology 15 (12): 1434-44.

Zhou, You-Bing, Chris Newman, Wen-Ting Xu, Christina D. Buesching, Andrzej Zalewski, Yayoi Kaneko, David W. Macdonald, and Zong-Qiang Xie. 2011. "Biogeographical Variation in the Diet of Holarctic Martens (genus Martes, Mammalia: Carnivora: Mustelidae): Adaptive Foraging in Generalists." Journal of Biogeography 38 (1): 137-47. 